\title{
Resolving the clumpy circumstellar environment of the $B[e]$ supergiant LHA 120-S 35
}

\author{
A. F. Torres ${ }^{1,2}$, L. S. Cidale ${ }^{1,2}$, M. Kraus ${ }^{3,4}$, M. L. Arias ${ }^{1,2}$, R. H. Barbá ${ }^{5}$, G. Maravelias ${ }^{3,6}$, and M. Borges Fernandes ${ }^{7}$ \\ ${ }^{1}$ Instituto de Astrofísica de La Plata (CCT La Plata - CONICET, UNLP), Paseo del Bosque S/N, La Plata B1900FWA, Buenos Aires, \\ Argentina \\ e-mail: atorres@fcaglp.unlp.edu.ar \\ 2 Departamento de Espectroscopía, Facultad de Ciencias Astronómicas y Geofísicas, Universidad Nacional de La Plata, Paseo del \\ Bosque S/N, La Plata B1900FWA, Buenos Aires, Argentina \\ ${ }^{3}$ Astronomický ústav, Akademie věd České republiky, Fričova 298, 25165 Ondřejov, Czech Republic \\ 4 Tartu Observatory, Tõravere, 61602 Tartumaa, Estonia \\ 5 Departamento de Física y Astronomía, Universidad de La Serena, Av. Cisternas 1200 Norte, La Serena, Chile \\ ${ }^{6}$ Instituto de Física y Astronomía, Universidad de Valparaíso, Av. Gran Bretaña 1111, Casilla 5030, Valparaíso, Chile \\ 7 Observatório Nacional, Rua General José Cristino 77, 20921-400 São Cristovão, Rio de Janeiro, Brazil
}

Received 5 August 2017 / Accepted 1 December 2017

\begin{abstract}
Context. $\mathrm{B}[\mathrm{e}]$ supergiants are massive post-main-sequence stars, surrounded by a complex circumstellar environment where molecules and dust can survive. The shape in which the material is distributed around these objects and its dynamics as well as the mechanisms that give rise to these structures are not well understood.

Aims. The aim is to deepen our knowledge of the structure and kinematics of the circumstellar disc of the B[e] supergiant LHA 120-S 35.

Methods. High-resolution optical spectra were obtained in three different years. Forbidden emission lines, that contribute to trace the disc at different distances from the star, are modelled in order to determine the kinematical properties of their line-forming regions, assuming Keplerian rotation. In addition, we used low-resolution near-infrared spectra to explore the variability of molecular emission. Results. LHA 120-S 35 displays an evident spectral variability in both optical and infrared regions. The P-Cygni line profiles of H I, as well as those of Fe II and O I, suggest the presence of a strong bipolar clumped wind. We distinguish density enhancements in the P-Cygni absorption component of the first Balmer lines, which show variations in both velocity and strength. The P-Cygni profile emission component is double-peaked, indicating the presence of a rotating circumstellar disc surrounding the star. We also observe line-profile variations in the permitted and forbidden features of Fe II and O I. In the infrared, we detect variations in the intensity of the H I emission lines as well as in the emission of the CO band-heads. Moreover, we find that the profiles of each [Ca II] and [O I] emission lines contain contributions from spatially different (complete or partial) rings. Globally, we find evidence of detached multiring structures, revealing density variations along the disc. We identify an inner ring, with sharp edge, where [Ca II] and [O I] lines share their forming region with the $\mathrm{CO}$ molecular bands. The outermost regions show a complex structure, outlined by fragmented clumps or partial-ring features of Ca II and O I. Additionally, we observe variations in the profiles of the only visible absorption features, the He I lines.

Conclusions. We suggest that LHA 120-S 35 has passed through the red-supergiant (RSG) phase and evolves back bluewards in the Hertzsprung-Russell diagram. In this scenario, the formation of the complex circumstellar structure could be the result of the windwind interactions of the post-RSG wind with the previously ejected material from the RSG. The accumulation of material in the circumstellar environment could be attributed to enhanced mass-loss, probably triggered by stellar pulsations. However, the presence of a binary companion cannot be excluded. Finally, we find that LHA 120-S 35 is the third B[e] supergiant belonging to a young stellar cluster.
\end{abstract}

Key words. stars: individual: LHA 120-S 35 - supergiants - stars: peculiar - stars: massive - circumstellar matter - Magellanic Clouds

\footnotetext{
${ }^{\star}$ Based on data acquired using (1) the du Pont Telescope at Las Campanas Observatory, Chile, under the programme CNTAC 200802 (PI: Barbá), (2) the MPG 2.2-m Telescope at La Silla Observatory, Chile, under the programme ID.: 094.A-9029(D) and under the agreement MPI-Observatório Nacional/MCTIC, Prog. ID.: 096.A-9030(A), (3) the J. Sahade 2.15-m Telescope at Complejo Astronómico El Leoncito, operated under agreement between the Consejo Nacional de Investigaciones Científicas y Técnicas de la República Argentina and the National Universities of La Plata, Córdoba and San Juan, (4) the 8.1-m Telescope at Gemini South Observatory, which is operated by the Association of Universities for Research in Astronomy, Inc.,
}

under a cooperative agreement with the NSF on behalf of the Gemini partnership: the National Science Foundation (United States), the National Research Council (Canada), CONICYT (Chile), the Australian Research Council (Australia), Ministério da Ciěncia, Tecnologia e Inovacão (Brazil) and Ministerio de Ciencia, Tecnología e Innovación Productiva (Argentina), under the programme GS-2013B-Q-6 (PI: L. Cidale), (5) the Southern Astrophysical Research (SOAR) telescope, which is a joint project of the Ministério da Ciência, Tecnologia, e Inovação (MCTI) da República Federativa do Brasil, the U.S. National Optical Astron. Obs. (NOAO), the University of North Carolina at Chapel Hill (UNC), and Michigan State University (MSU) and (6) the ESO Science Archive Facility. 


\section{Introduction}

The importance of studying massive stars in astrophysics is evident as they play an important role in the galactic evolution. These stars may show strong mass-loss rates, which affect their own evolution, as well as the chemistry and dynamics of their surrounding medium. Sometimes the released material is accumulated forming circumstellar envelopes with particular physical conditions that lead to the development of the $\mathrm{B}[\mathrm{e}]$ phenomenon. The mean properties of this phenomenon are the noticeable emission lines in the optical spectra of B-type stars, typically low-excitation permitted and forbidden transitions in neutral or low-ionized metals, and a strong infrared excess due to hot circumstellar gas and dust (Conti 1997). The B[e] phenomenon appears in many different stages of evolution, ranging from the pre-main-sequence to the planetary nebula or supergiant stages (Lamers et al. 1998).

Our interest is focused on $\mathrm{B}[\mathrm{e}]$ supergiant stars (B[e]SGs), that constitute a homogeneous group among evolved stars that show the $\mathrm{B}[\mathrm{e}]$ phenomenon. The study of this particular group can shed light on a short post-main-sequence evolutionary phase characterized by strong mass-loss rates. The ejected material of $\mathrm{B}$ [e]SGs shows two different regions: a fast polar wind structure and either a Keplerian or slow expanding equatorial disc or ring. The disc configuration has been confirmed by polarimetric and interferometric observations (Magalhaes 1992; Oudmaijer \& Drew 1999; Melgarejo et al. 2001; Magalhães et al. 2006; Domiciano de Souza et al. 2007; Millour et al. 2011; Cidale et al. 2012). These discs provide ideal conditions for molecule formation and dust condensation (Kraus et al. 2000; Stahl 2001).

The number of $\mathrm{B}[\mathrm{e}] \mathrm{SG}$ known nowadays is very small: two in M31, 15 in the Magellanic Clouds, and around 15 candidates in our Galaxy (Kraus 2009; Levato et al. 2014; Kraus et al. 2014), and although some of them are well studied many questions still remain unclear. So, an exhaustive research on a particular object can give us significant information about the physical properties of the circumstellar medium that could help in a near future to infer global properties of this particular group. To achieve this goal, we chose LHA 120-S 35, a poorly studied star of the Large Magellanic Cloud (LMC).

LHA 120-S 35 was identified as S 35 by Henize (1956) in his catalogue of $\mathrm{H} \alpha$ emission stars and nebulae. Gummersbach et al. (1995) classified this object as a B[e] supergiant and derived the following stellar parameters: $T_{\text {eff }}=22000 \mathrm{~K}, \log g=3.0$, $E(B-V)=0.06, L_{*}=1.6 \times 10^{5} L_{\odot}, R_{*}=28 R_{\odot}$, and a ZAMS mass of $M_{*} \sim 22 M_{\odot}$, based on the fitting of theoretical fluxes to the observed stellar continuum from the ultraviolet to the infrared. They also analysed a high-resolution optical spectrum and reported the presence of permitted and forbidden emission lines (of Fe II, Ti II, Cr II, [Fe II], and [S II]), as well as strong Balmer lies with complex P Cygni-type profiles. From archival observations of the Far Ultraviolet Spectrographic Explorer (FUSE), Penny \& Gies (2009) estimated the projected rotational velocity, $v \sin i=159 \mathrm{~km} \mathrm{~s}^{-1}$. Bonanos et al. (2009) presented its spectral energy distribution from $0.3 \mu \mathrm{m}$ to $24 \mu \mathrm{m}$, showing infrared features that confirmed the presence of dust. Infrared emission at $70 \mu \mathrm{m}$ was also detected. Recently, this star was included in the $K$-band SINFONI (Spectrograph for INtegral Field Observations in the Near Infrared) survey presented by Oksala et al. (2013), who reported the first detection of the $\mathrm{CO}$ band head emission at $2.3 \mu \mathrm{m}$. Assuming that $\mathrm{CO}$ molecules are located in a narrow ring around the star, they found from model fittings to the observed $\mathrm{CO}$ band head emission a $\mathrm{CO}$ column number density of $N_{\mathrm{CO}} \sim 2 \times 10^{21} \mathrm{~cm}^{-2}$ and a temperature of $T_{\mathrm{CO}} \sim 3000 \mathrm{~K}$. This temperature is much lower than the $\mathrm{CO}$ dissociation temperature $(5000 \mathrm{~K})$ and suggests that the material may be located in a detached disc structure (Liermann et al. 2010). Oksala et al. (2013) also noticed the presence of ${ }^{13} \mathrm{CO}$ lines and derived a ${ }^{12} \mathrm{C} /{ }^{13} \mathrm{C}$ ratio of around ten and interpreted that LHA 120-S 35 is close to entering the red supergiant phase or perhaps evolving bluewards after this phase.

In this work we report on the unpublished spectral appearance of LHA 120 -S 35 longwards to $5200 \AA$ which provides valuable additional information to the understanding of this peculiar object. The paper is organized as follows: in Sect. 2, we present our optical and infrared observations; in Sect. 3, we present an analysis of the main spectral features; in Sect. 4, we model the kinematics of the circumstellar material. In Sect. 5 we discuss our results in the context of possible scenarios and present our conclusions.

\section{Observations}

\subsection{Optical spectra}

Two high-resolution optical spectra of LHA 120-S 35 ( $R \sim 45000)$ were obtained on 15 November 2008, with the echelle spectrograph at the $2.5-\mathrm{m}$ du Pont Telescope at Las Campanas Observatory (LCO) in Chile. We selected the following configuration: a $1 \times 4$ arcsec slit and a Tek5 $2 \mathrm{k} \times 2 \mathrm{k}$ CCD detector, with a pixel size of $24 \mu \mathrm{m}$, and a $2 \times 2$-pixel binning. The spectral coverage ranges from $3600 \AA$ to $9200 \AA$. The exposure time was $2400 \mathrm{~s}$ for each spectrum, obtaining a signalto-noise ratio $(\mathrm{S} / \mathrm{N})$ per pixel in the $5500 \AA$ region of $\sim 60$. Data were reduced using standard $\mathrm{IRAF}^{1}$ tasks. Spectra were bias subtracted, flat-field normalized, and wavelength calibrated.

Four high-resolution optical spectra of LHA 120-S 35 were acquired on 3 December 2014 and 4 December 2015 (two spectra per night) with the Fiber-fed Extended Range Optical Spectrograph (FEROS), attached to the MPG/ESO 2.2-m telescope at La Silla Observatory, Chile. FEROS is a bench-mounted echelle spectrograph, which provides data with a resolving power $R \sim 48000$ and a spectral coverage from $3600 \AA$ to $9200 \AA$. An EEV $2 \mathrm{k} \times 4 \mathrm{k}$ CCD detector with a pixel size of $15 \mu \mathrm{m}$ was used. The exposure time of each spectrum was $1300 \mathrm{~s}$. The spectra were added to achieve a better S/N. The final S/N was $\sim 30$ per pixel in the $5500 \AA$ region. The reduction process was performed using the FEROS standard on-line reduction pipeline.

An additional pipeline-processed 1-D spectrum was retrieved from the ESO Science Archive Facility, which was obtained with FEROS on 26 November 2015. The exposure time was $2000 \mathrm{~s}$ and the final $\mathrm{S} / \mathrm{N}$ was $\sim 11.5$ per pixel in the $5500 \AA$ region.

A standard star was observed in each night of 2008 and 2014 for telluric correction, which was performed using standard IRAF tasks. For the FEROS data from 2015 a telluric template from another night was applied.

We complemented these observations with an echelle spectrum $(R \sim 12600)$ taken with a REOSC spectrograph attached to the 2.15-m telescope at the Complejo Astronómico El Leoncito (CASLEO), Argentina, on 27 November 2012. The adopted instrumental configuration was a $316 \mathrm{l} / \mathrm{mm}$ grating, a $350 \mu \mathrm{m}$ slit width, and a Tek $1 \mathrm{k} \times 1 \mathrm{k}$ CCD detector, binned in $2 \times 2$. The spectrum covers the wavelength range 5800-9100 A.

\footnotetext{
1 IRAF is distributed by the National Optical Astronomy Observatories, which are operated by the Association of Universities for Research in Astronomy, Inc., under cooperative agreement with the National Science Foundation.
} 
A. F. Torres et al.: Resolving the clumpy circumstellar environment of the B[e] supergiant LHA 120-S 35
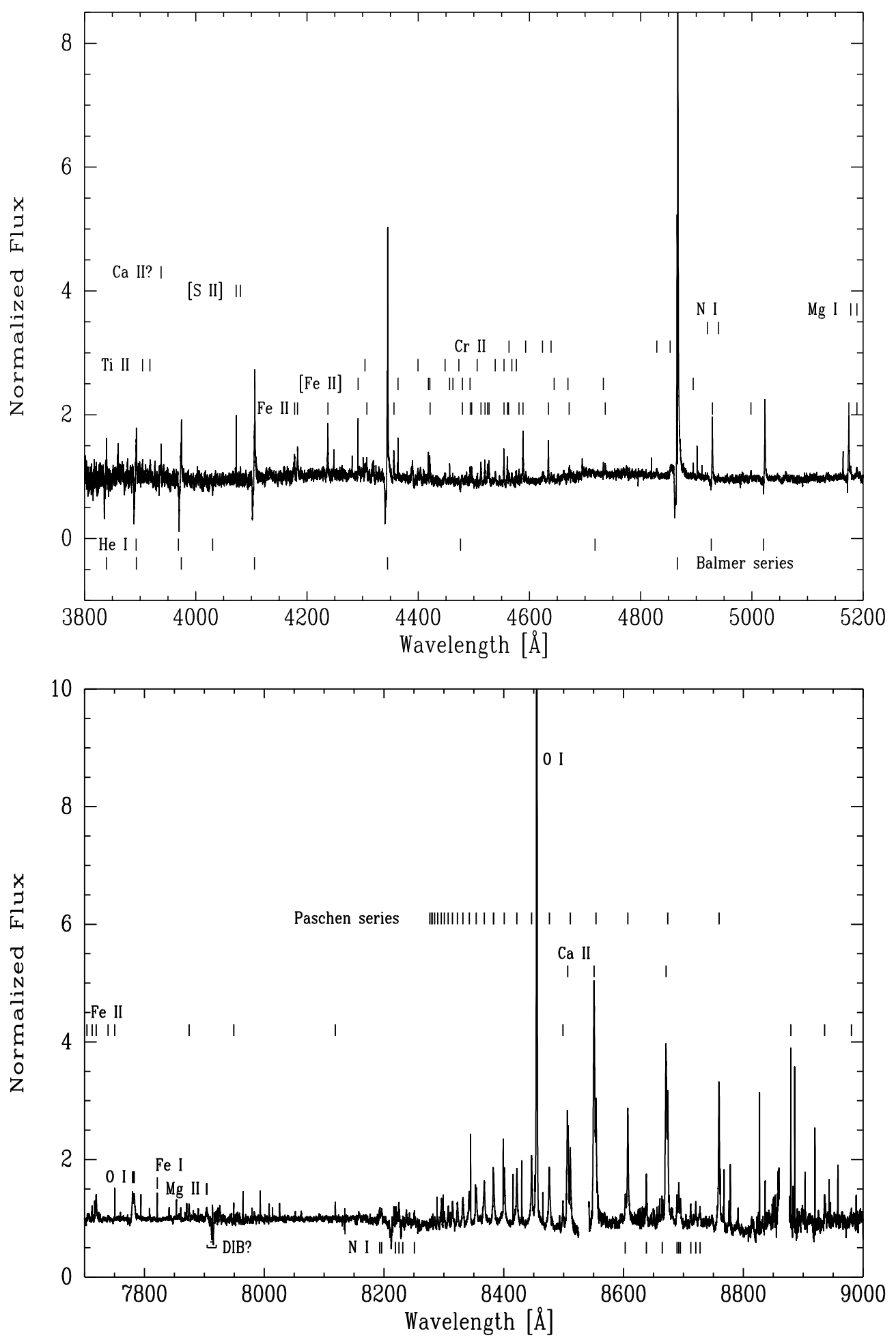

Fig. 1. FEROS spectrum from 2014 covering 3800-5200 A. Main emission lines are indicated. He I lines are in absorption.
Fig. 2. FEROS spectrum from 2014 covering $7700-9000 \AA$ A. Main emission lines are indicated. Two absorption features are visible, one could be attributed to a diffuse interstellar band (DIB) while the other remains unidentified.
The exposure time was $2100 \mathrm{~s}$ with a resulting $\mathrm{S} / \mathrm{N}$ per pixel of $\sim 6$ in the region around $6000 \AA$. We followed a standard reduction process using IRAF tasks. As the $\mathrm{S} / \mathrm{N}$ and the resolution of the CASLEO spectrum are considerably lower than those from the other spectra, we decided to include in this work only the $\mathrm{H} \alpha$ region.

\subsection{Near infrared spectra}

High-quality low resolution spectra $(R \sim 1200, \mathrm{~S} / \mathrm{N} \sim 100)$ of LHA $120-\mathrm{S} 35$ were taken in the $H K$ bands $(12500-25000 \AA)$ using the FLAMINGOS-2 spectrograph on the 8.1-m telescope at the Gemini South Observatory (Cerro Pachón, Chile) on 21 November 2013. The spectra were taken in the longslit mode using the $\mathrm{HK}_{G} 0806$ filter with the $\mathrm{HK}_{G} 5802$ grism centered at $1.871 \mu \mathrm{m}$. In order to remove the sky background, an ABBA nodding along the slit was required. A cycle of four ABBA sequences was performed. Immediately after, the late B-type star HIP 24337 (close to the target in position and airmass) was observed for telluric absorption correction. Late B- or early A-type standards are generally selected because the only intrinsic features they exhibit are neutral hydrogen lines. Flats were also acquired for flat fielding. The data were reduced with IRAF/Gemini tasks. We generated a telluric template to correct 

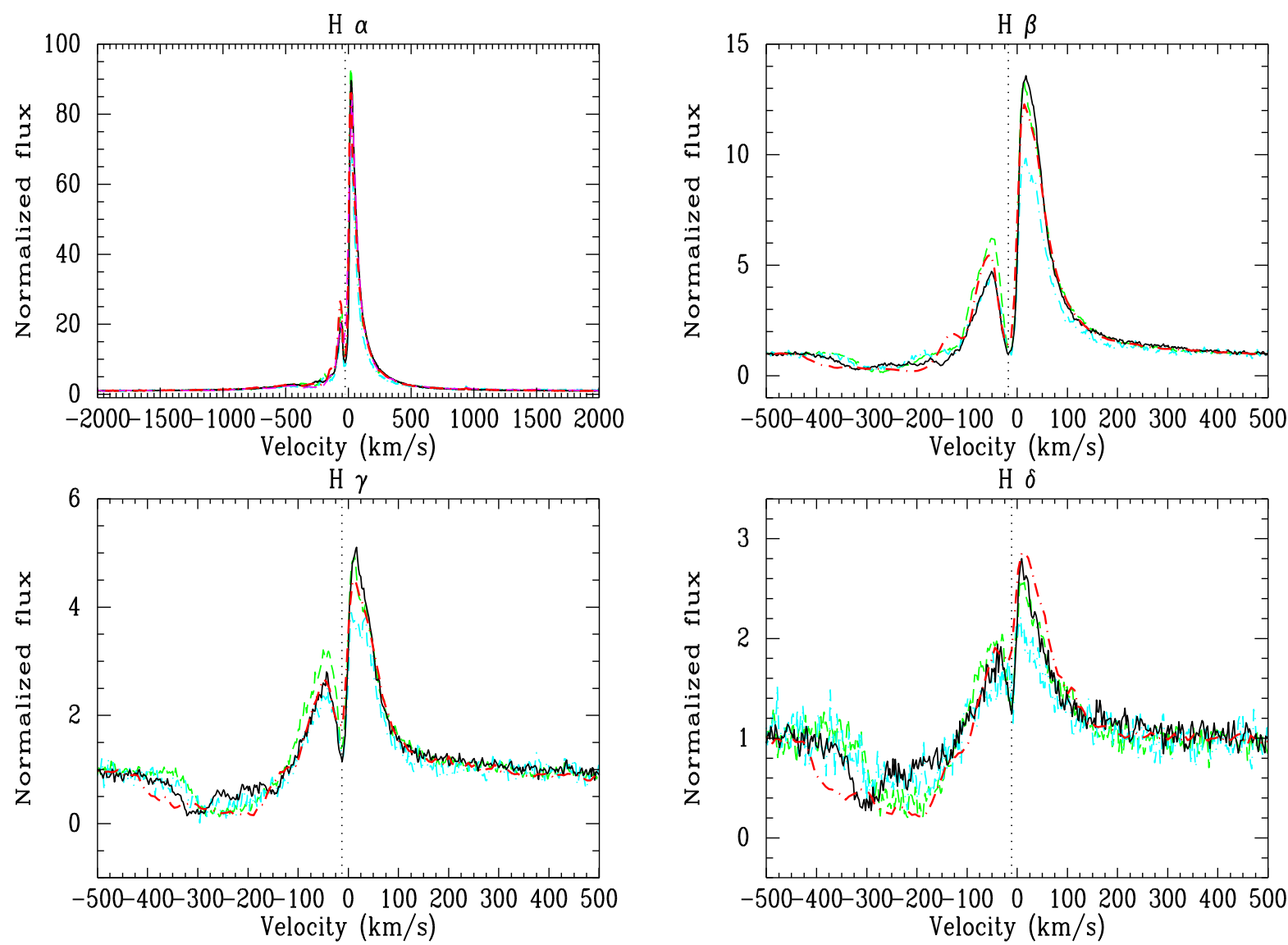

Fig. 3. Very complex P-Cygni profiles of the first Balmer lines seen in the spectrum of LHA 120-S 35 on a velocity scale relative to $V_{\text {sys }}=308 \mathrm{~km} \mathrm{~s}^{-1}$. The spectrum in red dash-dotted line was taken at LCO in 2008, while the ones in black solid, cyan dashed and green dash-dotted lines correspond to the observations acquired at ESO in 2014, November 2015 and December 2015, respectively. In the H $\alpha$ plot, we also included the spectrum acquired at CASLEO in 2012 in magenta dashed line. This colour coding will be used in the rest of the plots. The central absorption of the double-peaked emission component of the $\mathrm{H} \alpha, \mathrm{H} \beta, \mathrm{H} \gamma$ and $\mathrm{H} \delta$ lines is at $\sim 25 \mathrm{~km} \mathrm{~s}^{-1},-17 \mathrm{~km} \mathrm{~s}^{-1},-13 \mathrm{~km} \mathrm{~s}^{-1}$ and $-11 \mathrm{~km} \mathrm{~s}^{-1}$, respectively (indicated by a vertical dotted line).

the science stellar spectrum using the telluric IRAF task. The spectra were wavelength calibrated using the standard telluric star.

An additional observation was secured on 22 November 2012, with OSIRIS (the Ohio State InfraRed Imager/ Spectrometer) at the Southern Astrophysical Research (SOAR) 4.1-m telescope (Cerro Pachón, Chile). The spectrum was taken in cross-dispersion mode using the $\mathrm{f} / 2.8$ camera and a 1024 $\times 1024$ HAWAII HgCdTe array, covering the spectral range between 12500 and $23000 \AA$ with a low spectral resolution $(R \sim 1200)$. The reduction procedure was done with IRAF tasks, following the same steps than those already mentioned for FLAMINGOS-2 observations.

\section{Description of the observed main spectral features}

\subsection{In the optical}

LHA 120-S 35 presents a rich optical emission-line spectrum dominated by hydrogen lines as well as permitted and forbidden transitions of singly-ionized elements (such as Fe II, Ti II, S II, $\mathrm{Ca}$ II) and neutral atoms, like $\mathrm{O}$ I. The only absorption features are the He I lines. Figures 1 and 2 show the main spectral features in the ranges of 3800-5200 $\AA$ and 7700-9000 $\AA$, respectively.
The blue portion of the spectrum (3800-5200 ̊) resembles the one previously reported by Gummersbach et al. (1995). When comparing the same wavelength range (4450-4560 $\AA$ ) covered by our observations in 2008 and by Gummersbach et al. (1995, cf. their Fig. 2) we find no changes in shape and intensity for the main spectral features.

Hydrogen. Figure 3 shows the high-resolution spectra of the first Balmer lines $(\mathrm{H} \alpha, \mathrm{H} \beta, \mathrm{H} \gamma$ and $\mathrm{H} \delta$ ) taken with the echelle spectrograph of the du Pont telescope in 2008 and with FEROS in 2014, November 2015 and December 2015. The spectrum acquired at CASLEO in 2012 is included in the $\mathrm{H} \alpha$ plot. The most conspicuous feature of the whole spectrum is the $\mathrm{H} \alpha$ line, which shows a double-peaked emission profile with a deep central absorption component at $\sim-25 \mathrm{~km} \mathrm{~s}^{-1}$ and a violet-to-red ratio $V / R<1$, this value being smaller for the November 2015 spectrum. The red emission peak is at $\sim+21 \mathrm{~km} \mathrm{~s}^{-1}$ and the blue one at $\sim-61 \mathrm{~km} \mathrm{~s}^{-1}$, on a velocity scale relative to the systemic velocity $V_{\text {sys }}=+308 \pm 3 \mathrm{~km} \mathrm{~s}^{-1}$, which was derived by Gummersbach et al. (1995). A zoom of the $\mathrm{H} \alpha$ wings can be seen in Fig. 4. Three narrow absorption components (NACs) are observed on the blue wing of the emission line at around $-120 \mathrm{~km} \mathrm{~s}^{-1},-226 \mathrm{~km} \mathrm{~s}^{-1}$ and $-388 \mathrm{~km} \mathrm{~s}^{-1}$ in the du Pont spectrum and at around $-196 \mathrm{~km} \mathrm{~s}^{-1},-246 \mathrm{~km} \mathrm{~s}^{-1}$, and $-342 \mathrm{~km} \mathrm{~s}^{-1}$ in the FEROS spectrum from 2014 (indicated by arrows in Fig. 4), while in both spectra from 2015 only two dips are noticeable at around $-158 \mathrm{~km} \mathrm{~s}^{-1}$ and $-277 \mathrm{~km} \mathrm{~s}^{-1}$. In the 


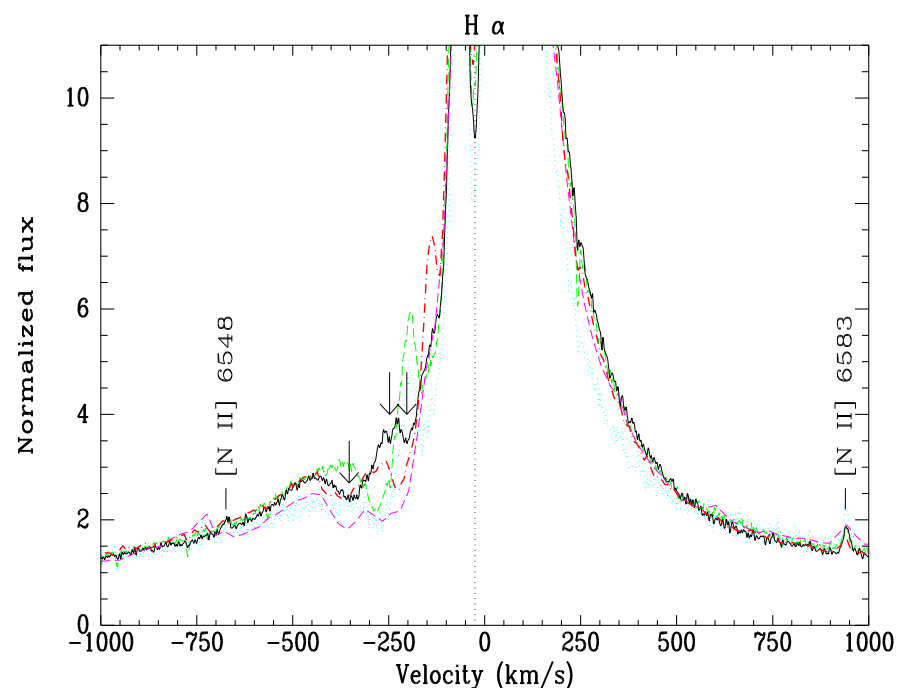

Fig. 4. Zoom of the very complex profile of the $\mathrm{H} \alpha$ line of LHA 120-S 35. A deep central absorption component at $\sim-25 \mathrm{~km} \mathrm{~s}^{-1}$ is indicated by a vertical dotted line. Narrow absorption components are marked with arrows on the 2014 FEROS spectrum. The [N II] $\lambda \lambda 6548$, 6583 lines are labelled. The same colour coding used in Fig. 3 is applied for Figs. 4-11.

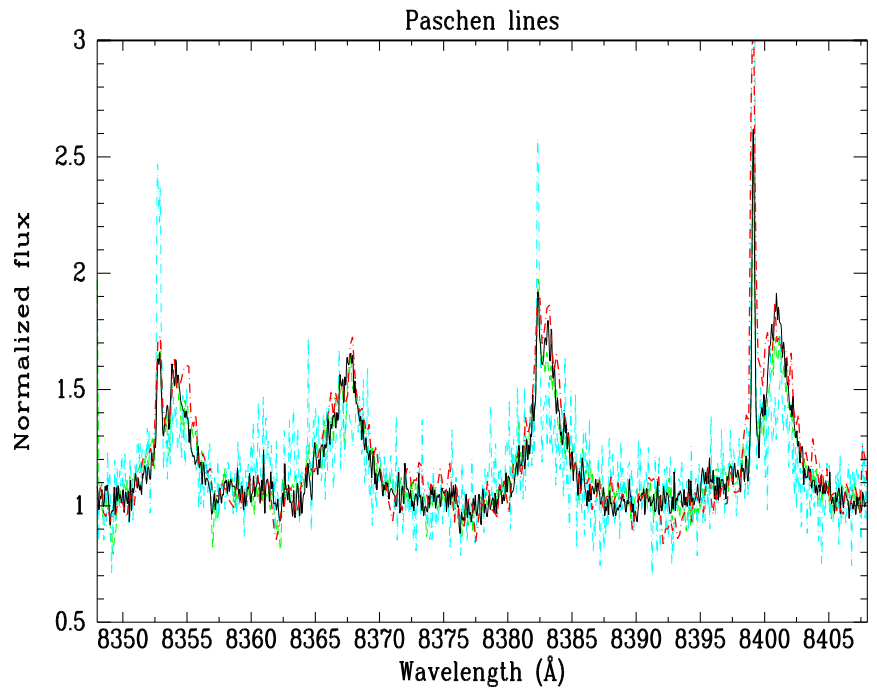

Fig. 5. Example of the Paschen series lines (Pa23, $\mathrm{Pa} 22, \mathrm{~Pa} 21$ and $\mathrm{Pa} 20$ lines, from left to right) of LHA 120-S 35. The sharp and narrow components seen on the left of the wide profiles could be related to the LMC background diffuse emission.

lower resolution CASLEO spectrum, two NACs are also clearly distinguishable at around $-267 \mathrm{~km} \mathrm{~s}^{-1}$ and $-359 \mathrm{~km} \mathrm{~s}^{-1}$. These features seem to move across the blue wing of the line.

The profiles of the rest of the first Balmer lines display a complex P-Cygni structure with its blue edge reaching a value of up to $-438 \mathrm{~km} \mathrm{~s}^{-1}$. Similar to the $\mathrm{H} \alpha$ line, the emission component presents two peaks at a mean wavelength of $\sim+12 \mathrm{~km} \mathrm{~s}^{-1}$ and $\sim-40 \mathrm{~km} \mathrm{~s}^{-1}$. The blue-shifted central absorption moves to higher velocities as we consider lower members of the series. In addition, NACs are detectable, especially in the spectrum from 2014, superimposed on the bottom of the profile's absorption trough of the $\mathrm{H} \beta$ line at $\sim-151 \mathrm{~km} \mathrm{~s}^{-1}$ and $\sim-322 \mathrm{~km} \mathrm{~s}^{-1}$ and of the $\mathrm{H} \gamma$ and $\mathrm{H} \delta$ lines at $\sim-143 \mathrm{~km} \mathrm{~s}^{-1}, \sim-223 \mathrm{~km} \mathrm{~s}^{-1}$ and $\sim-301 \mathrm{~km} \mathrm{~s}^{-1}$. The high
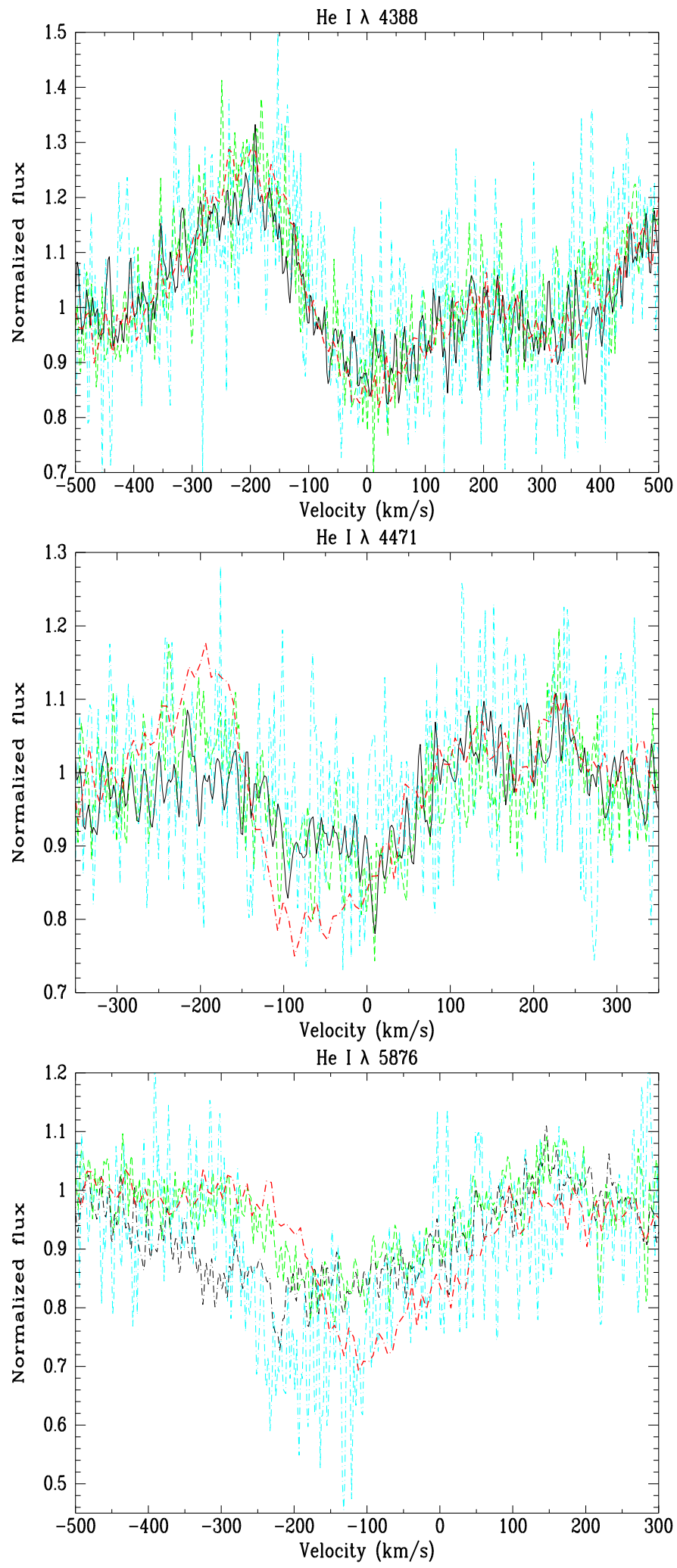

Fig. 6. Comparison of the shape of three He I lines of LHA 120-S 35 seen in the du Pont and FEROS spectra on a velocity scale relative to $V_{\text {sys. }}$. Changes in the blue wing of $\mathrm{He} \mathrm{I} \lambda \lambda 4471$ and 5876 lines are clearly observed.

members of the Balmer series exhibit typical P-Cygni features which are observed up to $\mathrm{H}_{22}$. Regarding the medium-resolution spectrum published by Gummersbach et al. (1995), the overall shape and intensity of the Balmer lines are the same as in the du Pont spectra. 

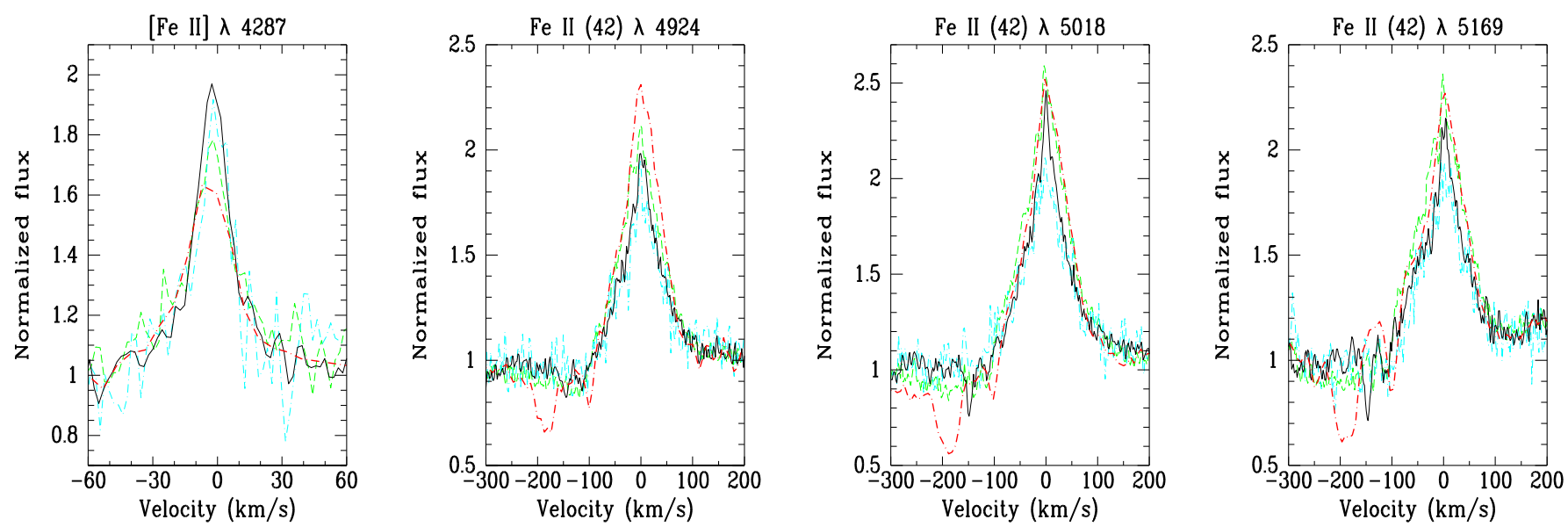

Fig. 7. Example of forbidden and permitted Fe II lines. The plots show the [Fe II] $\lambda 4287$ line and three permitted lines of the multiplet 42 at $\lambda 4924 \AA$ (blended with the He I $\lambda 4922$ line), $\lambda 5018 \AA$ (blended with the He I $\lambda 5016$ line) and $\lambda 5169 \AA$ on a velocity scale relative to $V_{\text {sys }}$. The multiplet 42 transitions display complex P-Cygni profiles, clearly seen in the du Pont and 2015 Nov FEROS spectra.

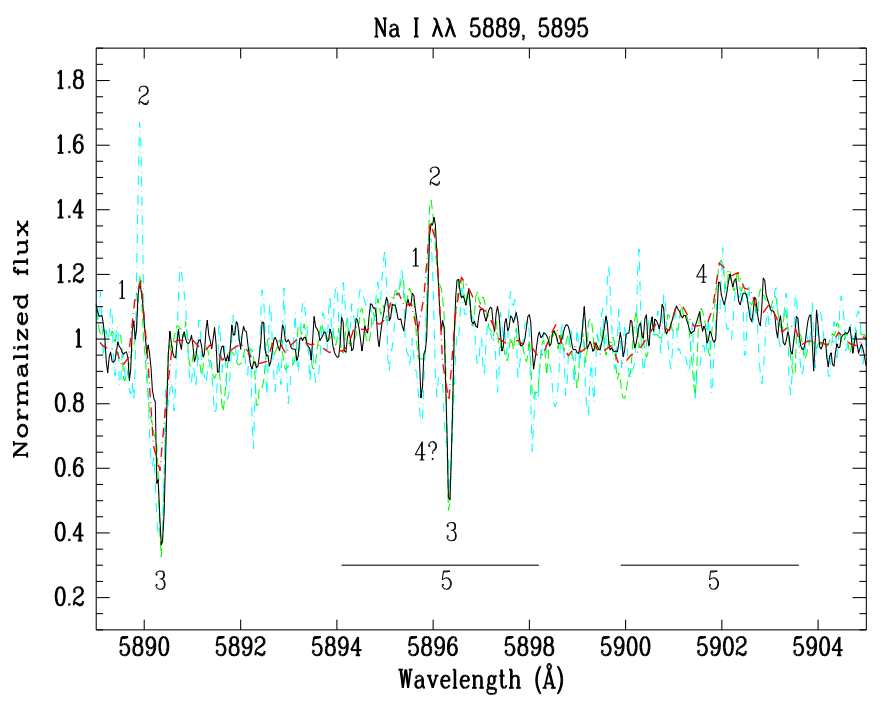

Fig. 8. Na I lines at $\lambda \lambda 5889,5895 \AA$ of LHA $120-\mathrm{S} 35$ seen in the du Pont and FEROS spectra. The doublet has different velocity components related to different formation regions (see text for more details about the labels).

Hydrogen Paschen lines are observed in pure emission up to $n=31$ with a velocity relative to $V_{\text {sys }}$ close to zero. The full width at half maximum (FWHM) of the lines ranges from $75 \mathrm{~km} \mathrm{~s}^{-1}$ to $165 \mathrm{~km} \mathrm{~s}^{-1}$, the spectrum from November 2015 presenting the widest lines. The line intensities in the spectrum from 2014 are higher than the ones from 2008 and 2015 (see Fig. 11), particularly the ones from November 2015 are the lowest. However, the intensities of the Paschen lines turn to be similar at the end of the series (see Fig. 5), indicating that these lines form in denser and, therefore, inner regions of the envelope.

Helium. The He I lines are in absorption. The He I $\lambda 4388$ singlet shows a complex structure (blended with the Ti II $\lambda 4387$ transition) and remains unchanged throughout all epochs, even in the poorest quality spectrum from November 2015 (where no global changes are observed whatsoever). Conversely, noticeable variations are observed in the line depth and the shape of the blue wing of He I $\lambda 4471$ and $\lambda 5876$ triplets (see Fig. 6). The He I $\lambda 4471$ line observed in the spectrum of the year 2008 resembles the one reported by Gummersbach et al. (1995), who identified the emission feature seen in the blue wing of the He I line as the Ti II $\lambda 4468$ transition. The change in the blue wing of the He I $\lambda 5876$ line profile is clearly detected in all spectra, even between the 2015 FEROS spectra obtained approximately one month apart. The He I $\lambda 4922$ and $\lambda 5016$ singlets are blended with the complex P-Cygni profiles of Fe II (42) multiplet (see Fig. 7), whose complex structure is blurred in the 2015 FEROS spectra. None of our spectra displays the He II $\lambda 4686$ line.

Iron. The spectrum is rich in both Fe II and [Fe II] emission lines. The FWHMs of Fe II and [Fe II] lines are about 85$105 \mathrm{~km} \mathrm{~s}^{-1}$ and $17-25 \mathrm{~km} \mathrm{~s}^{-1}$, respectively. In Fig. 7, the [Fe II] $\lambda 4287$ line and three Fe II transitions of multiplet 42 are shown. The last ones show complex P-Cygni features (clearly seen in the du Pont spectra and November 2015 FEROS spectra) with remarkable variations in the intensity of the absorption component.

Sodium. Several components are identified in the D1 and D2 lines (see Fig. 8). The absorption components 1 and 3 could be originated in the galactic interstellar medium (ISM). Their apparent complex feature could be the result of their blending with the night sky lines (components 2). Two broad components in emission (components 5) with a radial velocity similar to the systemic velocity, $\sim 308 \mathrm{~km} \mathrm{~s}^{-1}$, are also seen, probably arising from the circumstellar environment; their profiles do not show temporal variations. Finally, an absorption component (component 4 ) at $\sim 277 \mathrm{~km} \mathrm{~s}^{-1}$ is clearly detected superimposed to the second broad emission feature (D2 line). Since its radial velocity is close to the velocity of the LMC, this component 4 could be related to the LMC interstellar medium. The corresponding component 4 of the D1 line could be blended with the complex galactic ISM feature at $\lambda 5895 \AA$ (component 1$)$.

Oxygen. The spectra show prominent single-peaked emission lines of [OI] $\lambda \lambda 6300,6364 \AA$ with FWHMs of $\sim 24 \mathrm{~km} \mathrm{~s}^{-1}$ in the du Pont data and $\sim 19 \mathrm{~km} \mathrm{~s}^{-1}$ in the FEROS observations; these lines in the spectrum taken in 2014 are the most intense (see Fig. 9). The [O I] $\lambda 5577$ line is also visible and it seems not to show sensitive variations in strength (we excluded from the analysis the one corresponding to the November 2015 spectrum because it is very noisy). However, its FWHM ranges from $\sim 28 \mathrm{~km} \mathrm{~s}^{-1}$ in the FEROS spectrum from 2014 to $\sim 44 \mathrm{~km} \mathrm{~s}^{-1}$ in the rest of the spectra. The well-known 

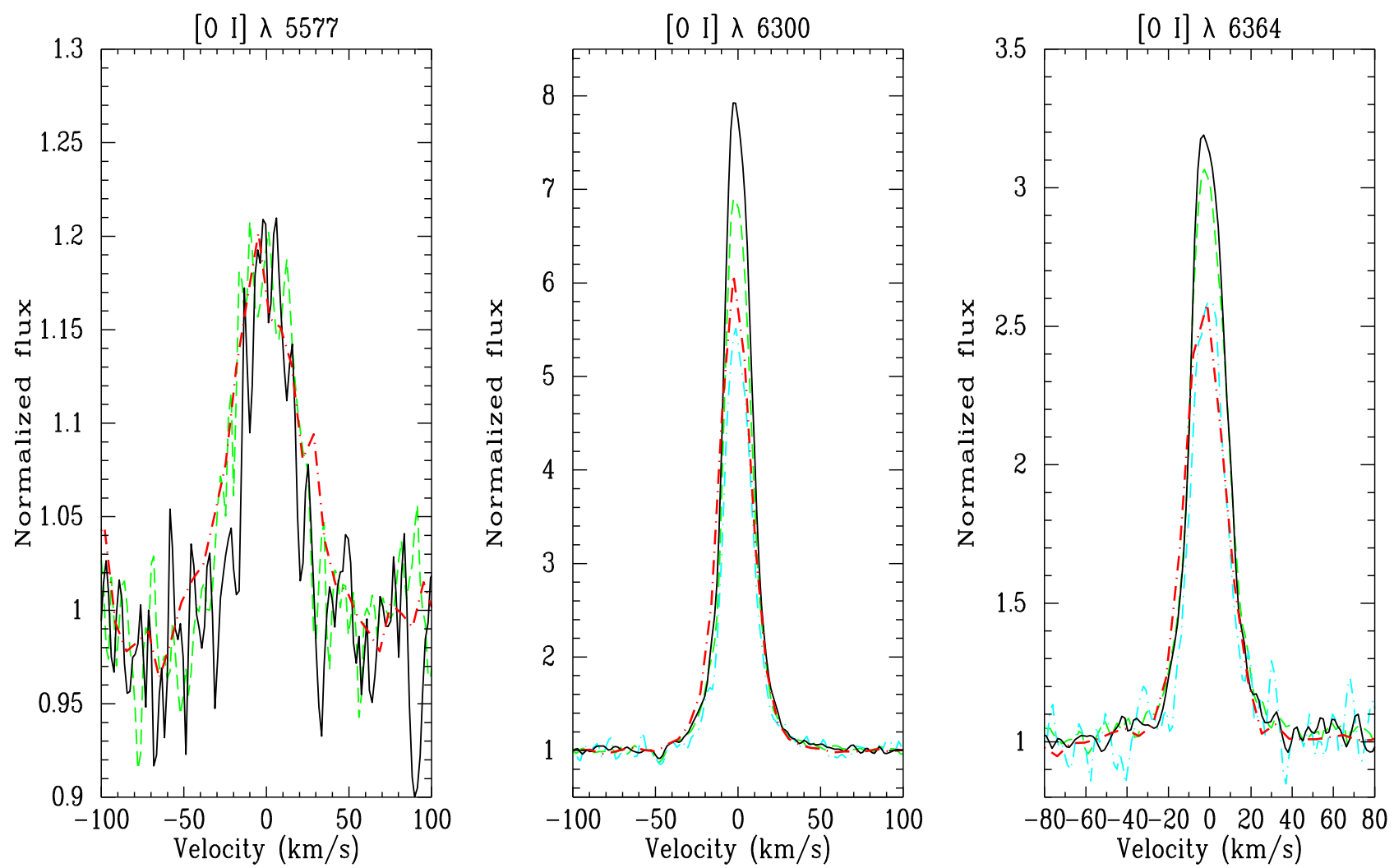

Fig. 9. Emission lines of [O I] at $\lambda \lambda$ 5577, 6300 and $6364 \AA$ of LHA 120-S 35 seen in the du Pont and FEROS spectra. We did not include in the analysis the [O I] $\lambda 5577$ line corresponding to the FEROS spectrum acquired in November 2015 because it is very noisy.

forbidden lines of O III at $\lambda \lambda 4959$ and $5007 \AA$ are absent. The strongest line of [O II] at $\lambda 7319 \AA$ is also not present.

In the red spectral region, the permitted O I $\lambda 8446$ triplet is the most remarkable emission feature, which could be attributed to a Ly $\beta$ fluorescence mechanism (Jaschek et al. 1993; Mathew et al. 2012). It is slightly blended with the Pa18 line ( $\lambda 8438 \AA$ ). Between the years 2008 and 2014, the line has increased its intensity by more than a factor of two, decreasing in the following year to about $40 \%$ of the maximum value and increasing again by more than $10 \%$ of this value in approximately one month. On the other hand, the O I triplet at $\lambda 7772-75 \AA$ shows a P-Cygni line profile with a weak emission. Figure 10 shows the variation in the P-Cygni absorption component of the triplet, which in the FEROS spectra of 2014 and November 2015 appears partially refilled by emission.

Calcium. The forbidden transitions of Ca II at $\lambda \lambda 7291$, $7324 \AA$ are clearly detected in emission. The [Ca II] $\lambda 7291$ line is variable and presents a double-peaked profile with a peak separation of $\sim 21 \mathrm{~km} \mathrm{~s}^{-1}$ from du Pont spectrum and an average of $\sim 33 \mathrm{~km} \mathrm{~s}^{-1}$ from FEROS spectra, with a notable width ranging from $\sim 110$ to $50 \mathrm{~km} \mathrm{~s}^{-1}$ in average, respectively (top left panel of Fig. 11). Since [Ca II] doublet arises in a region of strong telluric pollution, it is difficult to obtain reliable measurements, especially if the lines are weak and the tellurics cannot properly be removed; such is the case of the [Ca II] $\lambda 7324$ line (not shown here).

The near-infrared triplet of Ca II $\lambda \lambda 8498,8542,8662$ presents strong double-peaked emission features blended with the Paschen lines at $\lambda 8502 \AA, \lambda 8545 \AA$ and $\lambda 8665 \AA$, respectively (see Fig. 11). While hydrogen lines exhibit a noticeable intensity variation in 2014 with respect to the spectrum from 2008, the Ca II triplet shows a small intensity change, except for the weak calcium profiles registered in November 2015.

Nitrogen and sulphur. The [N II] $\lambda 5754$ and [N II] $\lambda 6584$ lines are also present in emission, but the [N II] $\lambda 6548$ line is only seen in the spectra acquired in 2014 and 2015 (see Fig. 4). The N III triplet at $\lambda \lambda 4634,4640,4642 \AA$ is absent in all our spectra. The transitions of [S II] at $\lambda \lambda 4069,4076 \AA$ are present in emission in all spectra as well as the transitions at $\lambda \lambda 6717$, $6731 \AA$, although the last ones are very weak.

Titanium oxide. From $\sim 6162 \AA$ to $6181 \AA$ a broad and weak emission feature can be detected in all epochs (see Fig. 12) with similar strength. In previous works a similar structure, clearly observed in four B[e]SGs belonging to the Magellanic Clouds, was associated to a TiO band emission (Zickgraf et al. 1989; Torres et al. 2012; Kraus et al. 2016).

\subsection{In the near-infrared}

$H K$-band spectra of LHA 120 -S 35 were taken with the OSIRIS and FLAMINGOS-2 spectrographs on November 2012 and 2013, respectively. The good-quality FLAMINGOS-2 spectrum displays the hydrogen $\mathrm{Pa} \alpha$ and $\mathrm{Pa} \beta$ lines in a prominent emission (see Fig. 13). The Brackett series lines are also visible in emission, up to the $n=22$ transition.

Moreover, the FLAMINGOS-2 spectrum shows the OI $\lambda 1.316 \mu \mathrm{m}$ and the Fe II $\lambda 1.687 \mu \mathrm{m}$ lines. A weak absorption feature is also observed near $2.058 \mu \mathrm{m}$ that corresponds to He I. Around the $\mathrm{Pa} \alpha$ line, the spectrum appears noisy, which could be related to a residual of the telluric correction process since at $1.9 \mu \mathrm{m}$ there is an intense telluric water band. In the same way, the broad emission feature at $1.36 \mu \mathrm{m}$ could be a result 

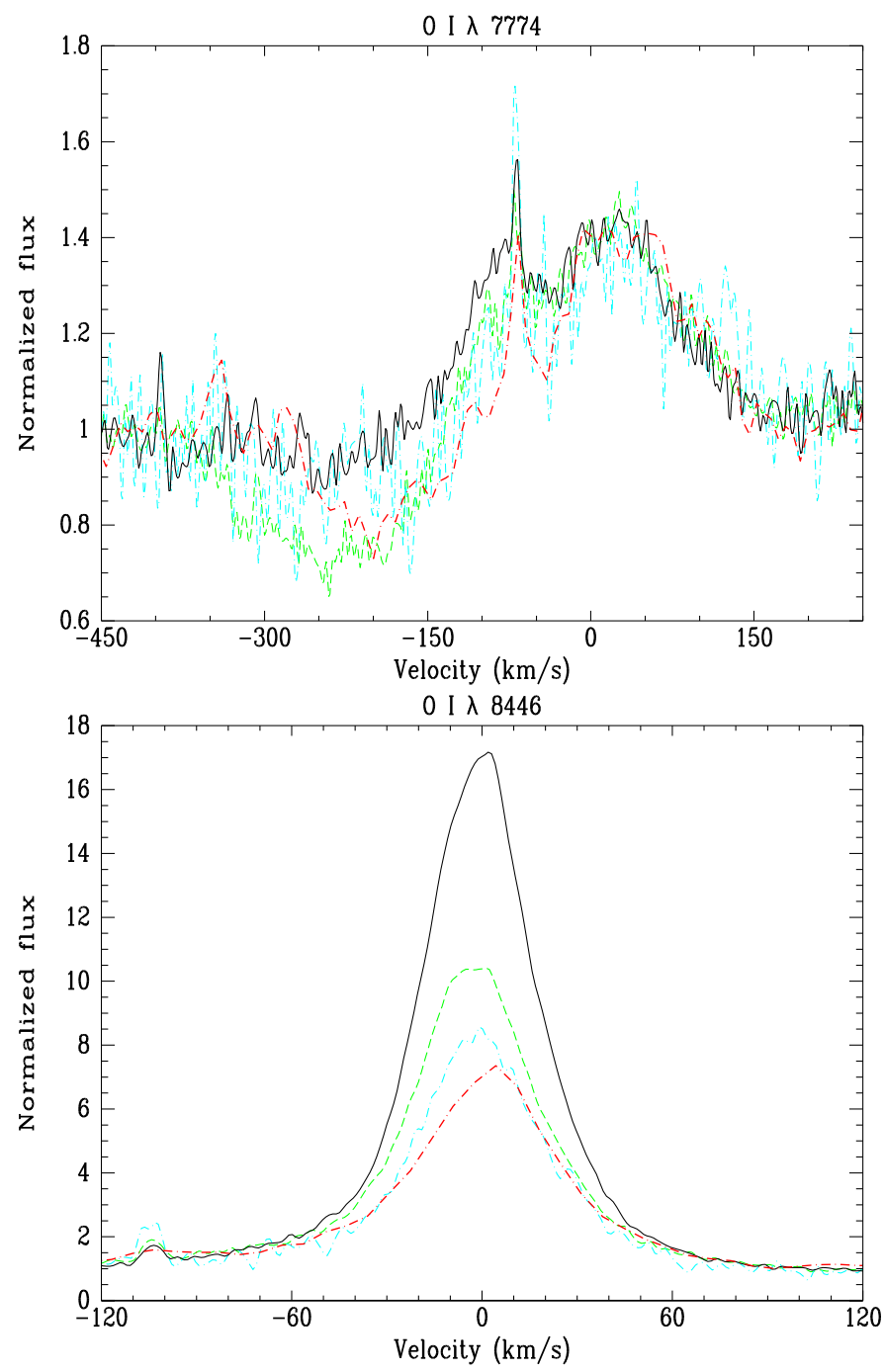

Fig. 10. Top panel: line profile of the O I $\lambda 7772-75$ triplet on a velocity scale relative to $V_{\text {sys. }}$. Bottom panel: comparison of the strength and shape of the permitted transition of O I $\lambda 8446$.

of the difficulty in removing the intense telluric band at $1.4 \mu \mathrm{m}$ completely.

Additionally, weak $\mathrm{CO}$ first overtone band emission is detected where the following ${ }^{12} \mathrm{CO}$ band heads can be identified: 2-0 (2.294 $\mu \mathrm{m}), 3-1(2.323 \mu \mathrm{m}), 4-2(2.352 \mu \mathrm{m}), 5-3(2.383 \mu \mathrm{m})$. Weak emission of the hydrogen Pfund series is observed in the $\mathrm{CO}$ band region as well.

Strong variability in the strength of the hydrogen lines and $\mathrm{CO}$ bands is detected when comparing the OSIRIS to the FLAMINGOS-2 spectra, taken almost one year later. Figure 14 shows that in spite of the poor quality of the OSIRIS spectrum the intensity of the Brackett lines is weaker than that observed in the spectrum of FLAMINGOS- 2 but conversely the $\mathrm{Pa} \beta$ line appears more than twice stronger. Regarding the $\mathrm{CO}$ bands in the OSIRIS spectrum, there does not seem to be any clear signs of emission. We cannot assert if the emission is either extremely weak or absent due to both the low resolution of the spectrum and the poor telluric correction beyond $2.2 \mu \mathrm{m}$ (see Fig. 14).

\section{Circumstellar gas variability}

We collected optical and near-IR spectra of high, moderate and low spectral resolution of LHA 120-S 35. All this information
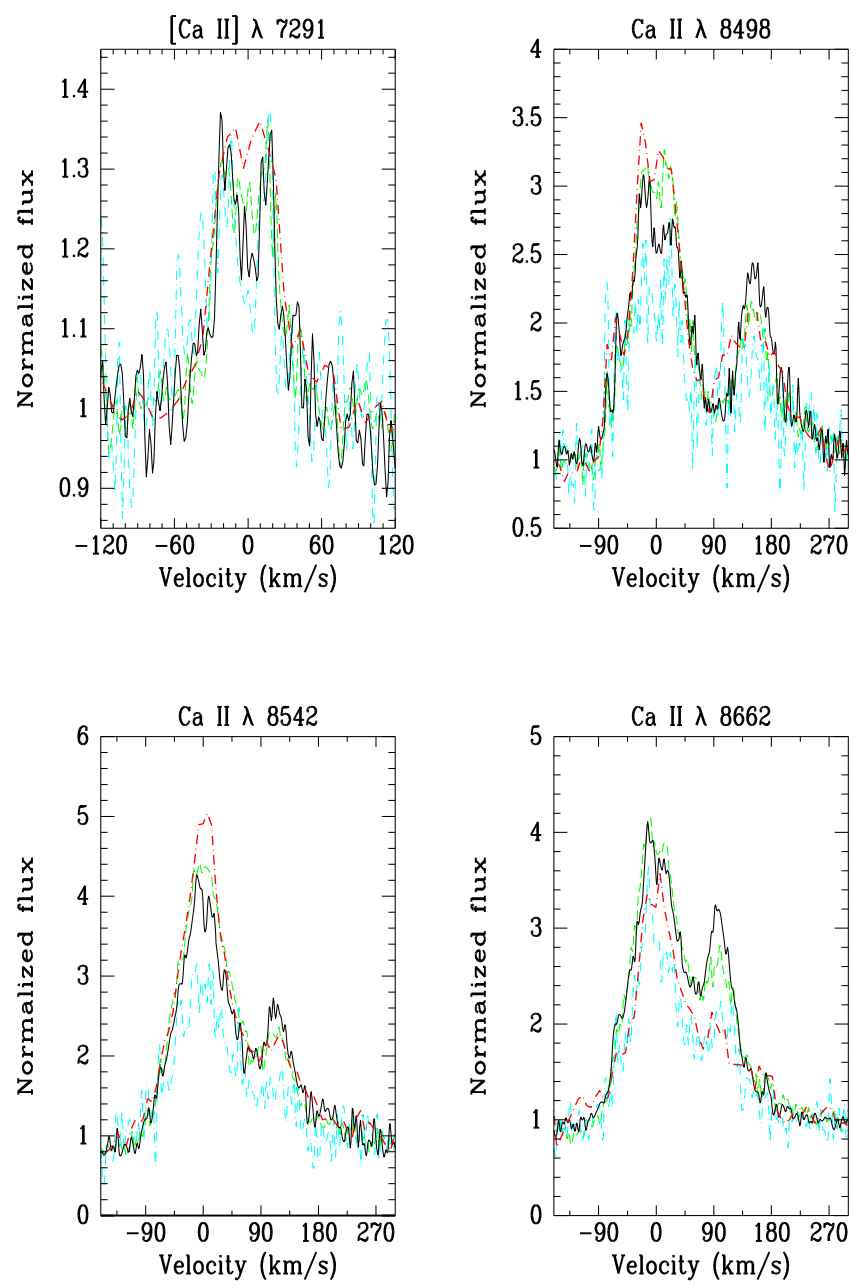

Fig. 11. Comparison of the strength and shape of the [Ca II] $\lambda 7291$ and the Ca II $\lambda \lambda 8498,8542,8662$ emission lines seen in the du Pont data and in FEROS spectra from 2014 and 2015. The Ca II triplet lines are blended with the Paschen lines Pa16, Pa15 and Pa13, respectively.

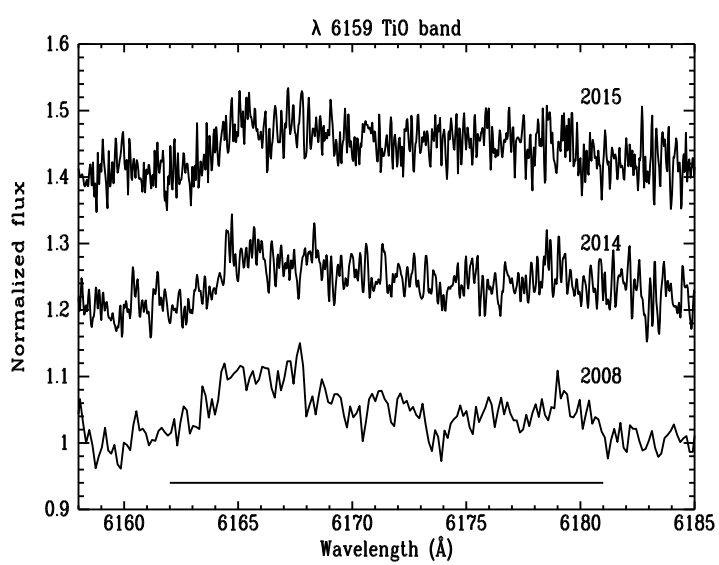

Fig. 12. Detection of a weak TiO band emission in the high-resolution optical spectra of LHA $120-\mathrm{S} 35$. The TiO feature from the November 2015 data is not included since it is very noisy.

allowed us to trace the kinematical properties of atomic and molecular gaseous emitting regions and discuss their variability. We assumed that the ejected mass from the star accumulates in a disc-like circumstellar structure and analysed its properties considering a Keplerian rotating disc. 


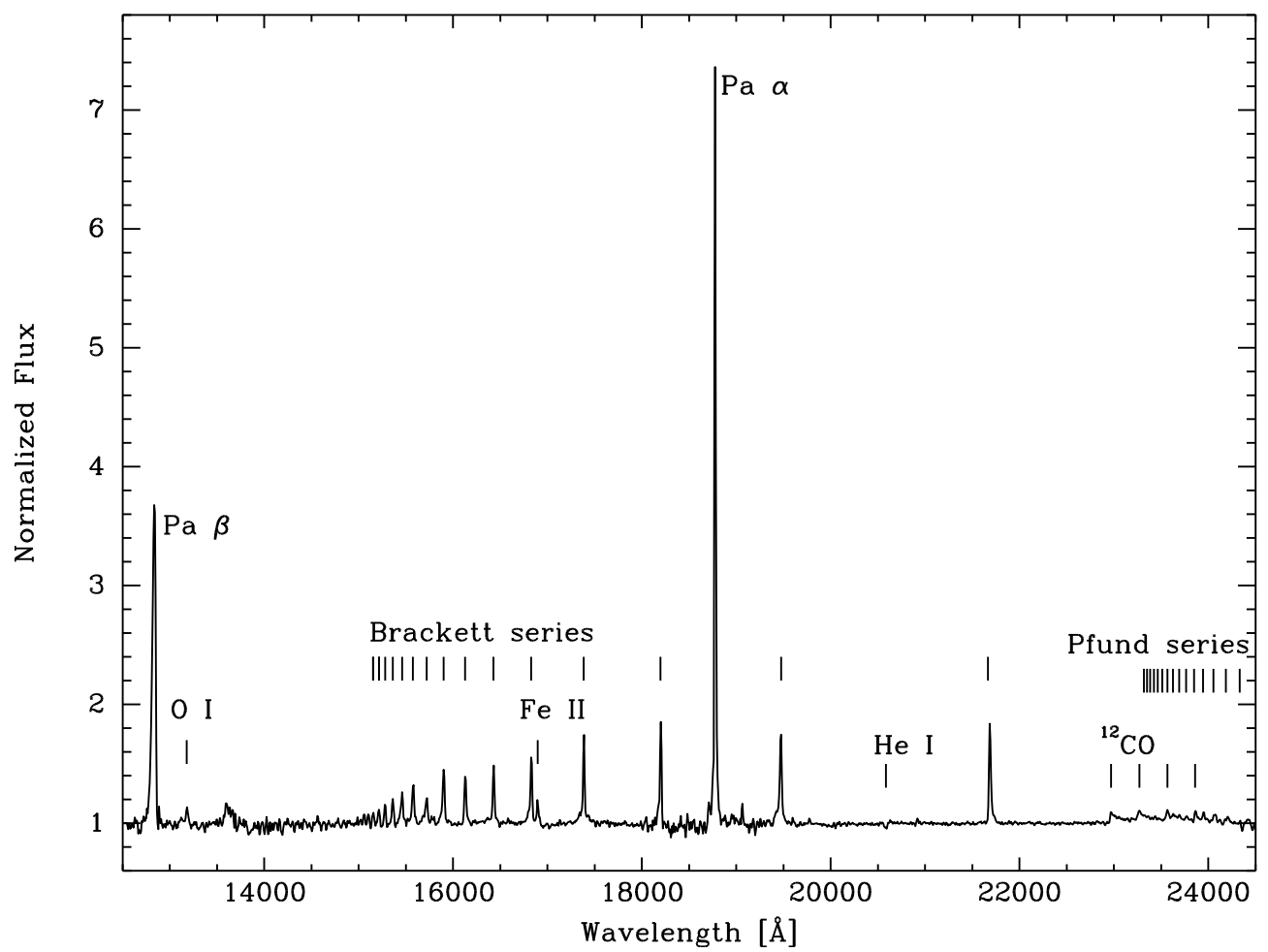

Fig. 13. Flux-normalized infrared spectrum of LHA 120-S 35 observed with the FLAMINGOS-2 spectrograph. The plot shows the $\mathrm{Pa} \beta$ and $\mathrm{Pa} \alpha$ prominent emission lines. The hydrogen Brackett series, the band heads of ${ }^{12} \mathrm{CO}$ and some other individual lines are indicated.

\subsection{The $C O$ band head emission}

The resolution of our near-IR spectra is too low to resolve the kinematics of the $\mathrm{CO}$ emitting region. However, to quantify the information of these data we proceeded in the following way. We considered the medium resolution SINFONI spectrum published by Oksala et al. (2013), in which a CO band head emission at $2.3 \mu \mathrm{m}$ was detected for the first time. This observation corresponds to February 2012 and was interpreted using a non-rotating disc model. However, generally, it is assumed that the disc formation around $\mathrm{B}[\mathrm{e}] \mathrm{SG}$ is related to fast rotation of the central stars. Thus, in order to improve Oksala et al.'s fit, we recomputed the physical parameters of the emitting $\mathrm{CO}$ gas assuming a rotating disc model. We applied the numerical codes developed by Kraus et al. (2000) and Kraus (2009). We considered that the $\mathrm{CO}$ emission arises from a thin ring in LTE in a rotating disc, where the ring temperature $T_{\mathrm{CO}}$, column density $N_{\mathrm{CO}}$ (along the line-of-sight), and rotational velocity $V_{\text {rot }}$ projected to the line-of-sight are constant. We computed both the ${ }^{12} \mathrm{CO}$ and ${ }^{13} \mathrm{CO}$ emission spectra. Additionally, the hydrogen Pfund series emission spectrum was modelled using the code developed by Kraus et al. (2000). We assumed the Pfund lines are optically thin and in LTE, which is valid if the emitting material originates either in a wind or a shell. We computed the spectral lines using a pure Gaussian profile with a line width of $30 \mathrm{~km} \mathrm{~s}^{-1}$ that then was convolved considering the SINFONI instrumental resolution. Figure 15 (left panel) displays the best-fit model to the SINFONI first overtone spectrum, which was obtained with the following physical parameters: $\mathrm{N}_{\mathrm{CO}}=2.5$ $\pm 0.5 \times 10^{21} \mathrm{~cm}^{-2}, \mathrm{~T}_{\mathrm{CO}}=2500 \pm 200 \mathrm{~K}, V_{\text {rot }}=35 \pm 5 \mathrm{~km} \mathrm{~s}^{-1}$, a microturbulent velocity of $V_{\text {turb }}=5 \pm 1 \mathrm{~km} \mathrm{~s}^{-1}$ and a ratio of ${ }^{12} \mathrm{C} /{ }^{13} \mathrm{C}=12.5 \pm 2.5$. These parameters are similar to the ones obtained by Oksala et al. (2013), although the temperature value is a bit smaller. However, we should emphasize that from medium and low resolution data, we can only determine an upper limit for the Keplerian projected rotational velocity of the $\mathrm{CO}$ gas. This estimation is the maximum possible value that $V_{\text {rot }}$ can reach in our computations before the theoretical spectrum begins to display features that are not observed in the SINFONI data.

Then, we convolved the best-fit $\mathrm{CO}$ model to the resolution of the FLAMINGOS-2 spectrum and overplotted it to the FLAMINGOS-2 data which were taken almost two years after the acquisition of the SINFONI spectrum done by Oksala et al. (2013). This comparison is shown in the middle panel of Fig. 15. Obviously, this model overestimates the observations. However, if we reduce the $\mathrm{CO}$ emission to $55 \%$ of its value in the SINFONI spectrum, without changing the Pfund emission, we reproduce the FLAMINGOS-2 $\mathrm{CO}$ band spectrum well enough (right panel). Therefore, we concluded that the intensity of the CO emission decreased in 2013, while the width of the band head seemingly did not change (at least not noticeable in our low-resolution spectra), which means that the rotational velocity of the emitting $\mathrm{CO}$ gas probably remained the same.

\subsection{Modelling optical forbidden emission lines}

One interesting property of the forbidden lines is that they are optically thin, hence their profiles carry the full kinematical information of their line-forming regions. Decoding this information from the [CaII] $\lambda 7291$ and [OI] $\lambda \lambda 5577,6300$ line profiles is useful to trace the ionized and neutral atomic disc regions close to the star. Previous investigations have shown that both the $\mathrm{CO}$ emission bands and the forbidden lines of $\mathrm{Ca}$ II and $\mathrm{O}$ I might share their forming regions, which can usually be described with a detached Keplerian rotating disc or ring model (Kraus et al. 2016; Maravelias et al. 2017). Therefore, to constrain the kinematical properties of the forbidden line-forming regions in LHA 120-S 35, we applied a purely kinematical model (Aret et al. 2016; Kraus et al. 2016), assuming that the emission originates from the same Keplerian rotating ring used in Sect. 4.1 to describe the $\mathrm{CO}$ emission bands. Thus, we computed a rotationally broadened line profile considering a projected 

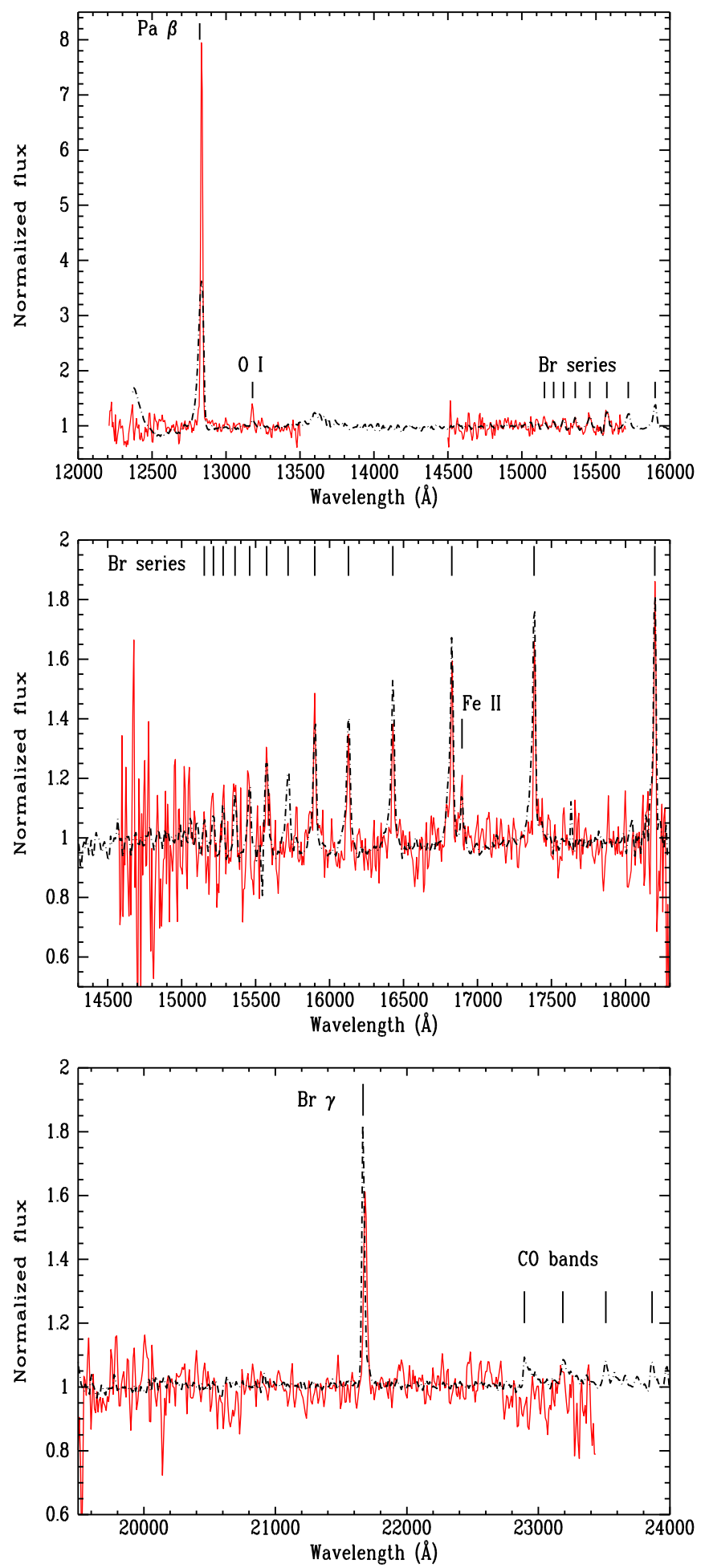

Fig. 14. Comparison of the flux-normalized infrared spectra of LHA 120-S 35 taken with the OSIRIS (in red solid line) and FLAMINGOS-2 (in dash-dotted black line) spectrographs. Despite of the poor S/N of the OSIRIS data, it can be seen that while the $\mathrm{Pa} \beta$ line is more than twice stronger than in the FLAMINGOS-2 spectrum, the Brackett series emission lines are weaker.

rotational velocity $V_{\text {rot }} \sim 35 \mathrm{~km} \mathrm{~s}^{-1}$. We convolved this velocity considering a Gaussian component, $V_{\text {gauss }}$, to model an extra broadening due to thermal and microturbulent motions of the gas (of a few $\mathrm{km} \mathrm{s}^{-1}$ ) and the instrument's spectral resolution.

If we compare the computed theoretical profile to the observed [Ca II] $\lambda 7291$ line profiles from both the du Pont and
FEROS spectra, we can see that the same (or a very similar) velocity component as for the $\mathrm{CO}$ ring seems to be present, as well as at least one more component that is required to fit the profiles properly (see Fig. 16). Therefore, to reproduce simultaneously the FWHM of the observed lines, the separation between peaks and the wings, we considered a multi-ring model composed by several concentric rings, each ring characterized by the following two parameters: $V_{\text {rot }}$ and $V_{\text {gauss }}$.

A good fit was obtained to the [Ca II] $\lambda 7291$ line profile for the du Pont spectra (see left panel of Fig. 17), considering a model composed by three individual gas rings with different rotational velocities. The same multi-ring model also fitted well the $[\mathrm{OI}]$ line profiles (see middle and right panels of Fig. 17), considering two individual rings with the sets of parameters given in Table 1, where we list $V_{\text {rot }}$ and $V_{\text {gauss }}$ in cols. 3 and 4, respectively.

The [O I] $\lambda 5577$ line profile from the FEROS observations (see Fig. 18) was also well reproduced by a multi-ring model consisting of two rings, but with different sets of parameters than the ones obtained for the du Pont profile fits. However, the theoretical profile resulting from a multi-ring model did not match the observed [CaII] $\lambda 7291$ line profiles from FEROS data. Likewise, the [O I] $\lambda 6300$ line profiles could not be fitted considering a model with homogeneous concentric rings, even by varying the number of rings and/or their rotational velocities. To solve these discrepancies, it was necessary to consider partial ring structures to describe the emitting region components. Figures 19 and 20 show the good fits obtained for the [O I] $\lambda 6300$ and [Ca II] $\lambda 7291$ line profiles, respectively. The best fitting parameters are given in Table 1, where we also list the shape of the ring components (col. 5), either complete or partial, indicating in the last case the angle subtended by each ring segment, and the minimum and maximum projected rotational velocities used as integration limits of each ring component (cols. 6 and 7, respectively).

We can summarize our results as follows. The gaseous equatorial disc where the forbidden emission lines of $\mathrm{O}$ I and $\mathrm{Ca}$ II (and the molecular emission of $\mathrm{CO}$ ) originate, shows regions of alternating density that form ring-like structures, either complete or fragmented. In some cases, the separation among these structures is not completely clear due to the large ring widths, since $V_{\text {gauss }}$ may reach some $\mathrm{km} \mathrm{s}^{-1}$ beyond spectral resolution. We highlight four main emitting regions in Keplerian rotation as constituents of our model.

From the fitting to the du Pont spectra, we can identify four major emitting rings with projected rotational velocities spanning the ranges $36-32 \mathrm{~km} \mathrm{~s}^{-1}, 22-20 \mathrm{~km} \mathrm{~s}^{-1}, 17-11 \mathrm{~km} \mathrm{~s}^{-1}$, and $3-1 \mathrm{~km} \mathrm{~s}^{-1}$. The [Ca II] $\lambda 7291$ emission line originates in the first three regions close to the star. The location of the [O I] $\lambda 5577$ line-forming regions coincides with the location of the first and third [Ca II] forming regions, while the [O I] $\lambda 6300$ line forms in the outermost two regions.

By fitting FEROS observations, we recognize a different disc spatial and kinematical configuration. The contribution of partial ring segments or fragmented clumps in Keplerian rotating regions is revealed. However, on comparing the modelling for both set of observations, we have to consider that the du Pont data were binned and thus have a lower resolution than the FEROS data. This may yield differences in the line profile shapes and thus different modelling results. Nevertheless, the possibility of a real change in the ring's structure might not be discarded.

The 2014 forbidden emission lines indicate the presence of four distinct emitting regions with projected rotational velocities of $\sim 37-35 \mathrm{~km} \mathrm{~s}^{-1}, \sim 23-21 \mathrm{~km} \mathrm{~s}^{-1}, \sim 18-9 \mathrm{~km} \mathrm{~s}^{-1}$ and 

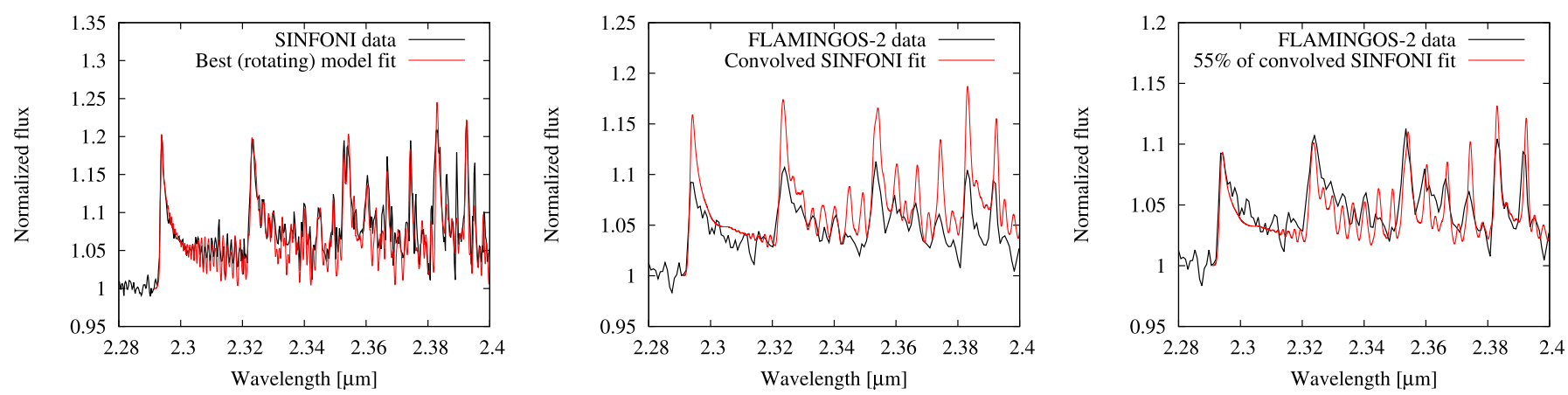

Fig. 15. Left panel: best model fit (in red) to the SINFONI CO band spectrum (in black), assuming a rotating CO ring scenario. Middle panel: comparison between the FLAMINGOS-2 CO band spectrum (in black) and the SINFONI's best model fit (in red). The synthetic spectrum has been convolved to FLAMINGOS-2 resolution. Right panel: comparison between the FLAMINGOS-2 CO band spectrum (in black) and the SINFONI's best model fit (in red), in which the emission was reduced to 55\% of its value in the SINFONI spectrum, while the Pfund emission was not altered.

$\sim 3-1 \mathrm{~km} \mathrm{~s}^{-1}$. Our results suggest that the inner forming-region of [Ca II] line is a complete ring, while the second one seems to be a ring segment. The third emitting region, where both $\mathrm{Ca}$ II and $\mathrm{O}$ I coexist, seems to present clumped structures. Finally, the outermost region, where the $\mathrm{O}$ I emission contributes to the forbidden transition at $\lambda 6300 \AA$, looks like a fragmented ring segment.

For the 2015 FEROS data, we obtain more or less the same distinct multi-ring structure, although for the November spectrum we only modelled the [O I] $\lambda 6300$ emission line, since the [O I] $\lambda 5577$ and the [Ca II] $\lambda 7291$ lines are distorted by the noise. We distinguish the first $\left(\sim 37-35 \mathrm{~km} \mathrm{~s}^{-1}\right)$ and third $(\sim 17-$ $\left.9 \mathrm{~km} \mathrm{~s}^{-1}\right)$ regions observed in 2014 . The second $\left(\sim 26-22 \mathrm{~km} \mathrm{~s}^{-1}\right)$ looks like an inhomogeneous ring with the coexistence of Ca II and $\mathrm{OI}$, which contributes to the [O I] $\lambda 5577$ emission. The fourth outermost partial ring $\left(\sim 3-1 \mathrm{~km} \mathrm{~s}^{-1}\right)$ gives rise, as before, to the $[\mathrm{O} I] \lambda 6300$ line.

The good matches between the computed profiles and the observed data showed a trend with respect to the multi-ring configuration: while the outermost rings are partial, the innermost are complete. It seems that the rings fragment or dissolve as they are getting older.

\section{Discussion and conclusions}

LHA 120-S 35 displays not only spectral variations but also photometric variability. Its $V$-band light curve, downloaded from the ASAS-3 Photometric $V$-band Catalogue (Pojmanski 2002), is displayed in Fig. 21. The data span a time interval of nine years, from November 2000 to November 2009. We checked the quality of each data point and kept only those that were labelled as quality $\mathrm{A}$. The final number of reliable measurements were thus reduced to 517 values. These are shown in Fig. 21 with their corresponding errorbars. We performed a period analysis with the AOV Periodogram Routines (Schwarzenberg-Czerny 1989, 1996; Schwarzenberg-Czerny \& Beaulieu 2006) and we did not find any reliable period to describe the variations. This could be due to the contamination of the photometry by a nearby star separated less than 12 arcsec, which is not resolved by the ASAS aperture photometry. However, a sort of long period $(P>1750 \mathrm{~d})$ oscillation may be present in the $V$-band light curve of LHA 120-S 35 on which there may be overlapping irregular light variations with amplitudes of about 0.2 mag, reaching up to $\sim 0.7 \mathrm{mag}$ in some sporadic points. Traditionally, it has been considered that $\mathrm{B}[\mathrm{e}] \mathrm{SG}$ show little or no photometric variations
[Ca II] $\lambda 7291$ - du Pont data

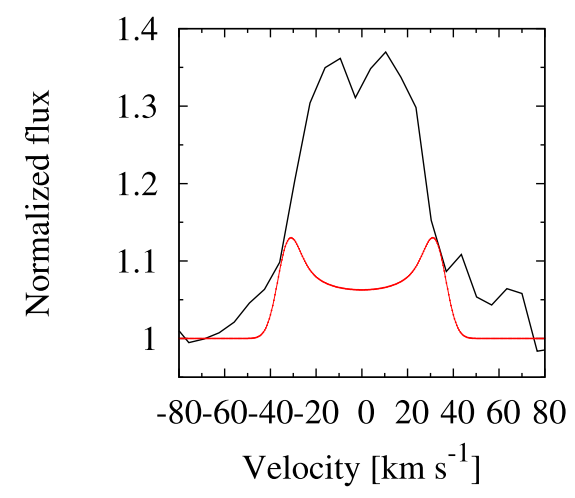

[Ca II] $\lambda 7291$ - 2014 FEROS data

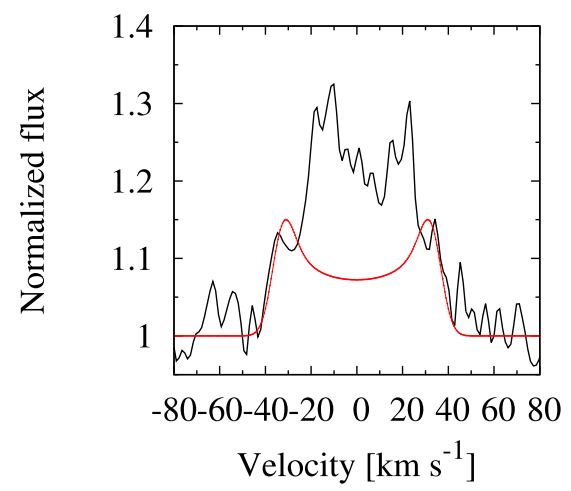

Fig. 16. Comparison between the observed [Ca II] $\lambda 7291$ line profile from the du Pont and 2014 FEROS spectra (top and bottom panels, respectively) and a computed theoretical profile considering a single ring model. Assuming the same rotational velocity as for the $\mathrm{CO}$ ring $\left(V_{\text {rot }}=35 \mathrm{~km} \mathrm{~s}^{-1}\right)$, the broadest part of the [Ca II] $\lambda 7291$ line profile could be reproduced (data shown in black line, and the model in red).

(Zickgraf et al. 1986; Lamers et al. 1998). However, the increase in the number of photometric observations not only has enlarged the observed B[e]SG sample but also has improved the temporal distribution of the data. From this, it was possible to find that the photometric behaviour of some $\mathrm{B}[\mathrm{e}] \mathrm{SG}$ disagrees with the historical picture as, for example, in LHA 115-S 18 which is highly variable on multiple timescales with amplitudes ranging from $\sim 0.1$ to $1.0 \mathrm{mag}$ (van Genderen \& Sterken 2002; Clark et al. 
[Ca II] $\lambda 7291$ - du Pont data

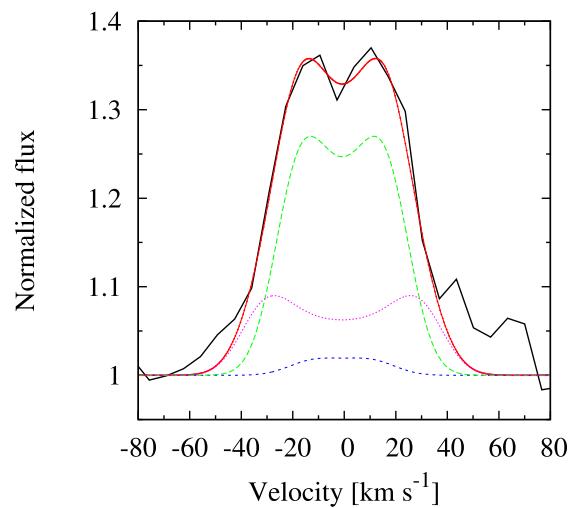

[O I] $\lambda 5577$ - du Pont data

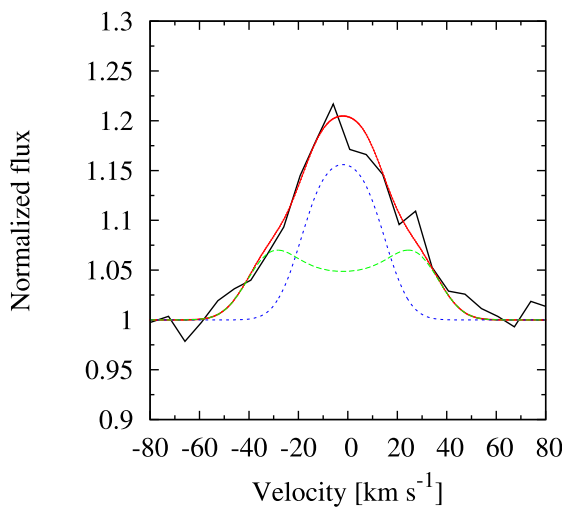

[O I] $\lambda 6300$ - du Pont data

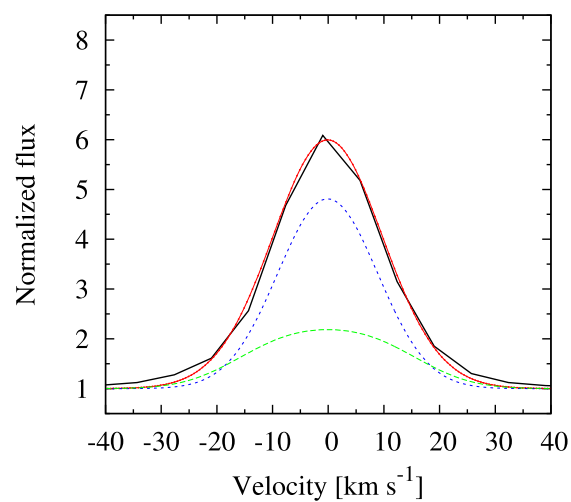

Fig. 17. Model fits to the [Ca II] $\lambda 7291$ (left panel), [O I] $\lambda 5577$ (middle panel) and [O I] $\lambda 6300$ (right panel) line profiles corresponding to the du Pont data. The computed profiles (in red lines) were obtained combining different emitting rings (in other colours) with different projected Keplerian rotational velocities (see Table 1).

Table 1. Best-fit model parameters to the forbidden line profiles for LHA 120-S 35.

\begin{tabular}{|c|c|c|c|c|c|c|}
\hline Spectra & Line & $\begin{array}{c}V_{\text {rot }} \\
\left(\mathrm{km} \mathrm{s}^{-1}\right)\end{array}$ & $\begin{array}{c}V_{\text {gauss }} \\
\left(\mathrm{km} \mathrm{s}^{-1}\right)\end{array}$ & Ring structure & $\begin{array}{c}V_{\text {rot }}^{\min } \\
\left(\mathrm{km} \mathrm{s}^{-1}\right)\end{array}$ & $\begin{array}{c}V_{\text {rot }}^{\max } \\
\left(\mathrm{km} \mathrm{s}^{-1}\right) \\
\end{array}$ \\
\hline \multicolumn{7}{|l|}{$2008 \mathrm{du}$ Pont } \\
\hline & {$[\mathrm{O} \mathrm{I}] \lambda 5577$} & $34.0 \pm 2$ & $12.5 \pm 2$ & Complete & -34.00 & 34.00 \\
\hline & & $13.0 \pm 2$ & $12.5 \pm 2$ & Complete & -13.00 & 13.00 \\
\hline & {$[\mathrm{OI}] \lambda 6300$} & $12.0 \pm 1$ & $12.5 \pm 1$ & Complete & -12.00 & 12.00 \\
\hline & & $2.0 \pm 1$ & $12.5 \pm 1$ & Complete & -2.00 & 2.00 \\
\hline & {$[\mathrm{Ca} \mathrm{II}] \lambda 7291$} & $34.0 \pm 1$ & $12.5 \pm 1$ & Complete & -34.00 & 34.00 \\
\hline & & $21.0 \pm 1$ & $12.5 \pm 1$ & Complete & -21.00 & 21.00 \\
\hline & & $16.0 \pm 1$ & $12.0 \pm 1$ & Complete & -16.00 & 16.00 \\
\hline \multicolumn{7}{|l|}{2014 FEROS } \\
\hline & {$[\mathrm{O} \mathrm{I}] \lambda 5577$} & $16.0 \pm 2$ & $12.5 \pm 2$ & Complete & -16.00 & 16.00 \\
\hline & & $11.0 \pm 2$ & $12.5 \pm 2$ & Complete & -11.00 & 11.00 \\
\hline & {$[\mathrm{OI}] \lambda 6300$} & $12.0 \pm 1$ & $12.5 \pm 1$ & Complete & -12.00 & 12.00 \\
\hline & & $10.0 \pm 1$ & $6.5 \pm 1$ & Partial $\left(90^{\circ}\right)$ & 0.00 & 10.00 \\
\hline & & $2.0 \pm 1$ & $6.5 \pm 1$ & Partial $\left(10^{\circ}\right)$ & -2.00 & -1.96 \\
\hline & {$[\mathrm{Ca}$ II $] \lambda 7291$} & $36.0 \pm 1$ & $12.5 \pm 1$ & Complete & -36.00 & 36.00 \\
\hline & & $22.0 \pm 1$ & $6.5 \pm 1$ & Partial $\left(170^{\circ}\right)$ & -22.00 & 21.66 \\
\hline & & $15.0 \pm 1$ & $9.5 \pm 1$ & Complete & -15.00 & 15.00 \\
\hline \multicolumn{7}{|l|}{ Nov 2015 FEROS } \\
\hline & {$[\mathrm{OI}] \lambda 5577$} & $\ldots$ & $\ldots$ & $\ldots$ & $\ldots$ & $\ldots$ \\
\hline & {$[\mathrm{OI}] \lambda 6300$} & $15.0 \pm 1$ & $12.5 \pm 1$ & Complete & -15.00 & -15.00 \\
\hline & & $10.0 \pm 1$ & $6.5 \pm 1$ & Partial $\left(90^{\circ}\right)$ & 0.00 & 10.00 \\
\hline & & $2.0 \pm 1$ & $6.5 \pm 1$ & Partial $\left(10^{\circ}\right)$ & -2.00 & -1.96 \\
\hline & {$[\mathrm{Ca}$ II $] \lambda 7291$} & $\ldots$ & $\ldots$ & $\ldots$ & $\ldots$ & $\ldots$ \\
\hline \multirow[t]{9}{*}{ Dec 2015 FEROS } & & & & & & \\
\hline & {$[\mathrm{O} \mathrm{I}] \lambda 5577$} & $24.0 \pm 2$ & $12.5 \pm 2$ & Complete & -24.00 & 24.00 \\
\hline & 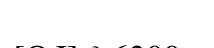 & $14.0 \pm 2$ & $12.5 \pm 2$ & Complete & -14.00 & 14.00 \\
\hline & {$[\mathrm{OI}] \lambda 6300$} & $16.0 \pm 1$ & $12.5 \pm 1$ & Complete & -16.00 & 16.00 \\
\hline & & $10.0 \pm 1$ & $6.5 \pm 1$ & Partial $\left(90^{\circ}\right)$ & 0.00 & 10.00 \\
\hline & & $2.0 \pm 1$ & $6.5 \pm 1$ & Partial $\left(10^{\circ}\right)$ & -2.00 & -1.96 \\
\hline & {$[\mathrm{Ca} \mathrm{II}] \lambda 7291$} & $34.0 \pm 1$ & $12.5 \pm 1$ & Complete & -34.00 & 34.00 \\
\hline & & $24.0 \pm 1$ & $6.5 \pm 1$ & Partial $\left(170^{\circ}\right)$ & -23.63 & 24.00 \\
\hline & & $16.0 \pm 1$ & $9.5 \pm 1$ & Complete & -16.00 & 16.00 \\
\hline 2012 SINFONI & CO bands & $\leq 35.0 \pm 5$ & $5.0 \pm 1$ & Complete & -35.00 & 35.00 \\
\hline
\end{tabular}

Notes. The last line shows the best-fit model parameters to the $\mathrm{CO}$ emission bands. 
[O I] $\lambda 5577$ - 2014 FEROS data

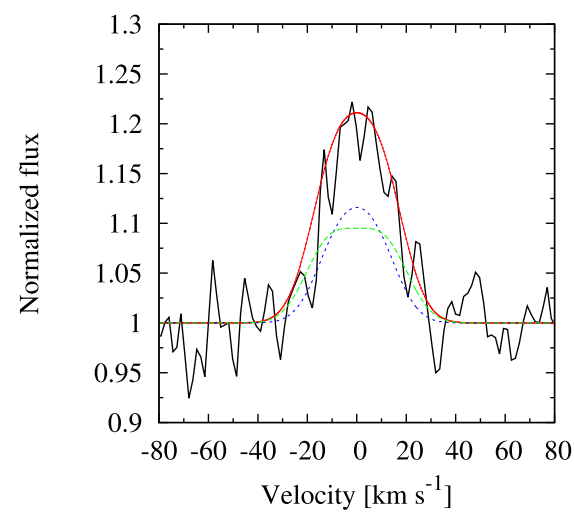

[O I] $\lambda 5577$ - Dec. 2015 FEROS data

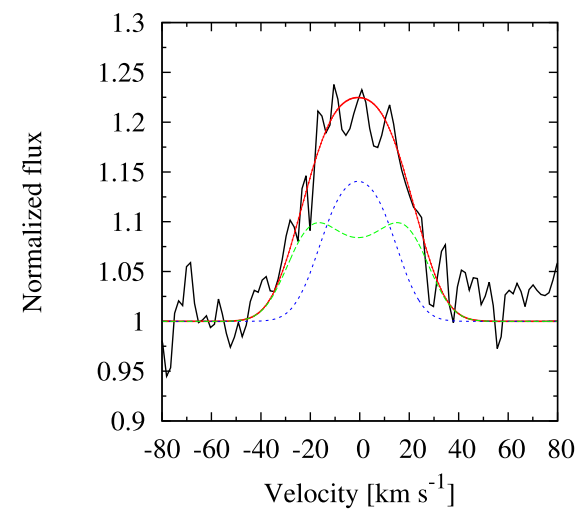

Fig. 18. Model fits to the [O I] $\lambda 5577$ line profiles corresponding to the FEROS data (2014 and December 2015 data, top and bottom pan$e l s$, respectively). The [O I] $\lambda 5577$ line from November 2015 FEROS spectra could not be modelled since it is very noisy.

2013a; Maravelias et al. 2014), the B[e]SG/X-ray binary CI Cam which showed evidence of variability both in the X-ray outburst and in quiescence (Clark et al. 2000), VFTS 698 with photometric variations of $\sim 0.6 \mathrm{mag}$ on timescales ranging from days to years (Dunstall et al. 2012) and MWC 349A with periodic variations of 0.6 mag in amplitude (Yudin 1996; Jorgenson et al. 2000). It is important to remark that all these stars are proposed or considered as members of binary systems (Clark et al. 2013a; Bartlett et al. 2013; Kim \& Taam 2012; Sallum et al. 2017) while the binary nature of LHA $120-\mathrm{S} 35$ is still inconclusive.

Spectroscopic variations are observed in both the optical and infrared wavelength ranges. Basically all optical lines display variabilities in shape and/or strength. The most pronounced changes are seen in the forbidden and permitted $\mathrm{O}$ I lines, these lines being more intense in 2014. Similarly, the [Fe II] lines show strong variability with the 2014 spectra displaying the highest intensity.

The spectroscopic analysis of LHA 120-S 35 suggests that the star has a strong and variable bipolar clumped wind, which is revealed throughout the P-Cygni line profiles of $\mathrm{HI}$, Fe II and O I. Density enhancements moving across the wind are seen superimposed to the absorption P-Cygni profiles (NACs) in the first Balmer lines at different velocities, showing variations in both intensity and velocity. NACs features in the optical lines were also seen in the luminous blue variables (LBVs). Groh \& Vink (2011) modelled the moving NACs seen in the LBV AGCar and explained their behaviours due to abrupt changes in the wind-terminal velocity, associated to the bi-stability jump.
This jump is related to a change in the degree of ionization in the wind (Vink 2000) which is caused by the recombination of Fe IV to Fe III. However, observations of early B SGs showed that there is a gradual decrease in the wind terminal velocities as a function of effective temperature, instead of a 'jump' (Crowther et al. 2006), and that the mechanism of bi-stability could be present among stars with temperatures ranging between $18000 \mathrm{~K}$ and $23000 \mathrm{~K}$ (Markova \& Puls 2008). The effective temperature of LHA 120-S 35 (22000 K, Gummersbach et al. 1995) falls into this range, so a similar phenomenology could take place in this star.

Additionally, the double-peaked features seen in the emission component of the Balmer P-Cygni profiles with the blue-shifted central absorption suggest the presence of a slow outflowing rotating circumstellar disc. A similar global structure composed by an equatorial disc perpendicular to a bipolar flow has been proposed to interpret the observations at millimeter and radio wavelengths for the B[e]SGs MWC 349 (Danchi et al. 2001; Tafoya et al. 2004) and Wd1-9 (Fenech et al. 2017) and the optical and infrared observations of MWC 137 (Kraus et al. 2017).

The shape of the Paschen lines seems to indicate a twocomponent forming region as well, a weak but broad, maybe double-peaked profile underneath a narrower single-peaked profile, where the first might come from the disc or rings and the second from the wind. Considering that intensity changes are only observed in the lower members of the Paschen series, which are formed in much outer regions than the higher members, they might be influenced by the clumpy structure of the rings.

In the outer part of this disc, we found spectroscopic evidences of detached multi-ring structures with density variations along the disc. The inner ring has a sharp edge where [Ca II] and $[\mathrm{O} \mathrm{I}]$ lines share their forming region with the $\mathrm{CO}$ molecular bands. The outermost regions show a complex structure, with fragmented clumps and/or partial-ring features traced by Ca II and $\mathrm{O}$ I. The shape of these complete and partial extended ring structures together with their dynamics (apparent expansion, fragmentation, re-formation at high velocities within short time scales) reminds of (spiral) density waves that can be excited in circumstellar discs via gravitational forces and which have been observed in planetary systems (e.g. Shu 2016 and references therein) and in protoplanetary discs (e.g. Fukagawa et al. 2004; Pérez et al. 2016). However, our observational data set is too sparse to confirm such a scenario and to identify its possible mechanism amongst which are the interaction with a companion, planet-disc interaction, or gravitational instabilities.

In order to interpret the spectroscopic variations of the forbidden lines, our model computations were performed assuming Keplerian (complete or partial) ring-like structures located in the equatorial plane. However, this scenario is not unique. The observed ring segments could be the result of the projection onto the sky plane of a three-dimensional structure (for example, an hourglass nebula) with density enhancements. If we consider that LHA 120-S 35 is an evolved object displaying a circumstellar environment that is oxygen-rich and contains a large amount of dust, the formation of such an hourglass-like structure might result from wind-wind interactions of the current wind of the star in its presumable post-red supergiant (post-RSG) state with previously ejected material from the RSG (Chita et al. 2008). However, in this case an hourglass-like structure can only be achieved if the wind is not spherically symmetric anymore. 
[O I] $\lambda 6300$ - 2014 FEROS data

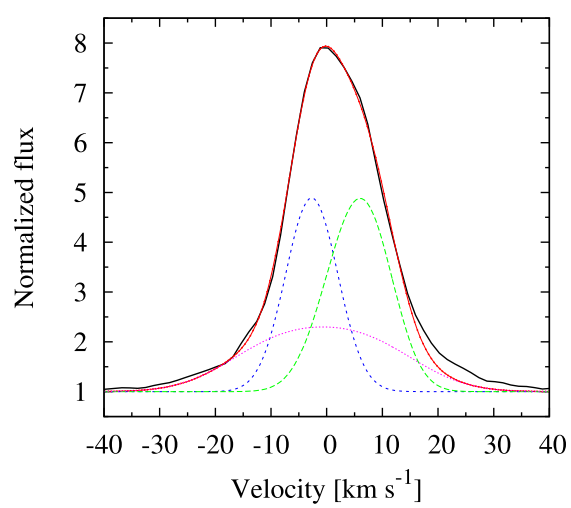

[O I] $\lambda 6300$ - Nov. 2015 FEROS data

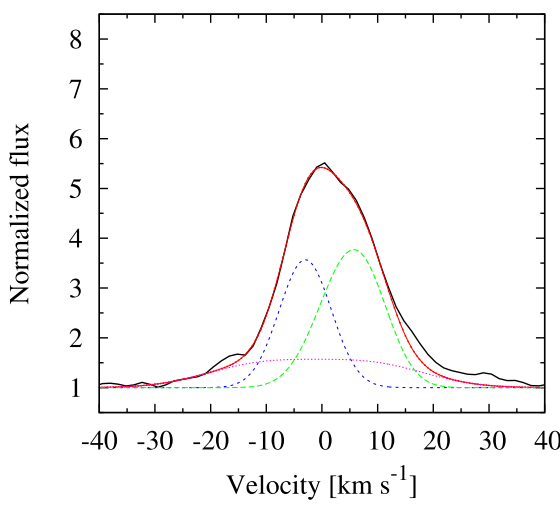

[O I] $\lambda 6300$ - Dec. 2015 FEROS data

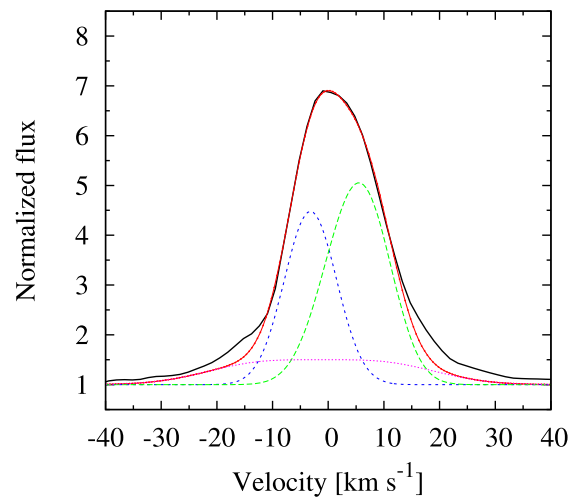

Fig. 19. Model fits to the [O I] $\lambda 6300$ line profiles corresponding to the FEROS spectra (2014, November 2015 and December 2015 data, left, middle and right panels, respectively). The computed profiles (in red line) were obtained combining different emission structures with different projected Keplerian rotational velocities (see Table 1).

[Ca II] $\lambda 7291$ - 2014 FEROS data

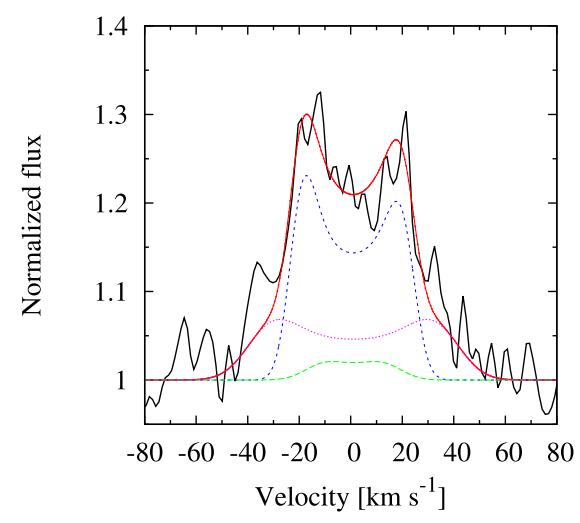

[Ca II] $\lambda 7291$ - Dec. 2015 FEROS data

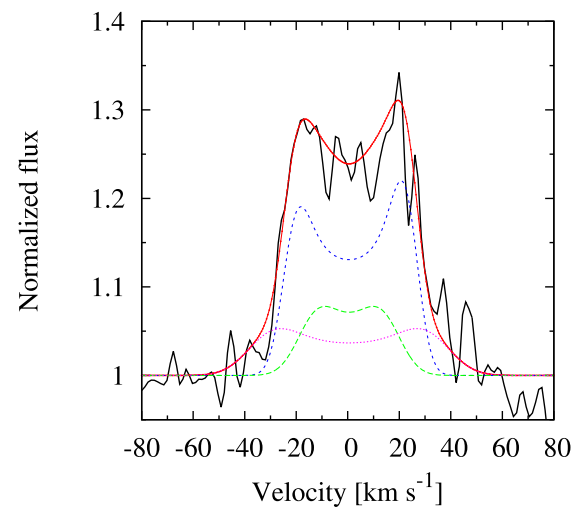

Fig. 20. Model fits to the [Ca II] $\lambda 7291$ profiles corresponding to the FEROS spectra (2014 and December 2015 data, top and bottom panels, respectively). The computed profiles (in red line) were obtained combining different emission structures with different projected Keplerian rotational velocities (see Table 1). The [Ca II] $\lambda 7291$ line from November 2015 FEROS data could not be modelled since it is very noisy.

Interestingly, Davies et al. (2007) suggested that the direct successors of RSGs, the yellow hypergiants (YHGs), change their mass-loss behaviour from spherical to axisymmetric during their passage through the instability domain called Yellow Void. This change results in the formation of an equatorial disc and a

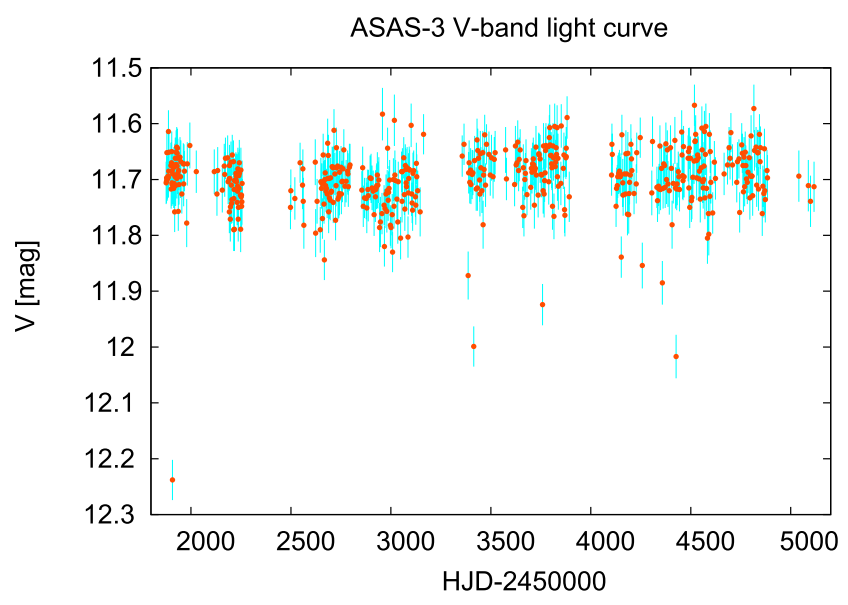

Fig. 21. ASAS-3 $V$-band light curve of LHA 120-S 35. Although no reliable period was found to describe the variations, a sort of long period $(P>1750 \mathrm{~d})$ oscillation may be present on which there may be overlapping irregular light variations with amplitudes of about 0.2 mag.

bipolar wind, and it is assumed that YHGs appear as B[e]SGs after they reach the blue edge of the instability domain (Davies et al. 2007; Aret et al. 2017). The YHGs pulsations are believed to trigger the enhanced mass-loss (de Jager 1998). Whether such a scenario also holds for the B[e]SGs cannot be firmly said. However, Kraus et al. (2016) recently postulated that the B[e]SG LHA 120-S 73 displays indications for pulsation activity based on the observed He I line profile variability. Inspection of the profiles of the He I $\lambda 5876$ line, which seems to be the only unblended absorption line in the optical spectrum of LHA 120S 35 reveals that this line also shows variability in its profile that might originate from stellar pulsations.

Alternatively, even though evidence indicative of binarity has not yet been found in LHA 120-S 35, a Roche lobe overflow phase in an interacting binary or a colliding-wind binary system could also be suitable scenarios to analyse the complex circumstellar ring-like (and possibly spiral) structures of LHA 120-S 35, as for example in RY Scuti (Smith et al. 2011) or Wd1-9 (Clark et al. 2013b). However, emission in key lines attributed to binarity as N III $\lambda \lambda 4634-42$ and He II $\lambda 4686$ is not seen, conversely to what was detected in $\mathrm{B}$ [e]SGs known to be binaries like LHA 115-S 18 (Clark et al. 2013a) and HD 38489 (Massey et al. 2014). The He II $\lambda 4686$ line is highly variable in 

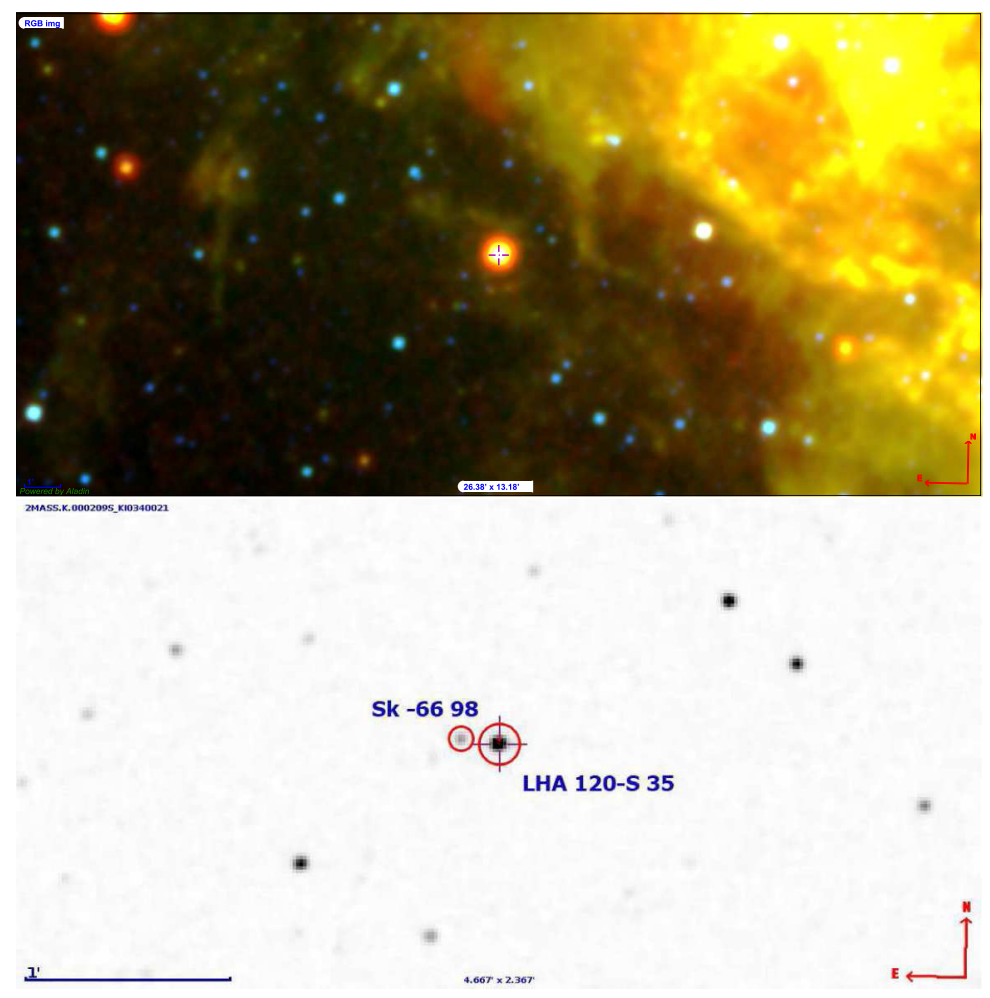

Fig. 22. Top panel: representative colour RGB WISE image $\left(\sim 26 \times 13 \mathrm{arcmin}^{2}\right)$ of LHA 120-S 35 and its surroundings made from images at $4.6 \mu \mathrm{m}$ (in blue), $12 \mu \mathrm{m}$ (in green) and $22 \mu \mathrm{m}$ (in red) with an angular resolution of $6.4,6.5$ and 12.0 arcsec, respectively. Bottom panel: 2MASS $K$-band image $\left(\sim 4.7 \times 2.4 \mathrm{arcmin}^{2}\right)$ showing LHA 120-S 35 (indicated by a cross) along with the neighbouring star Sk -6698 , which remains unresolved in the WISE image. Both images are orientated north up and east to the left.

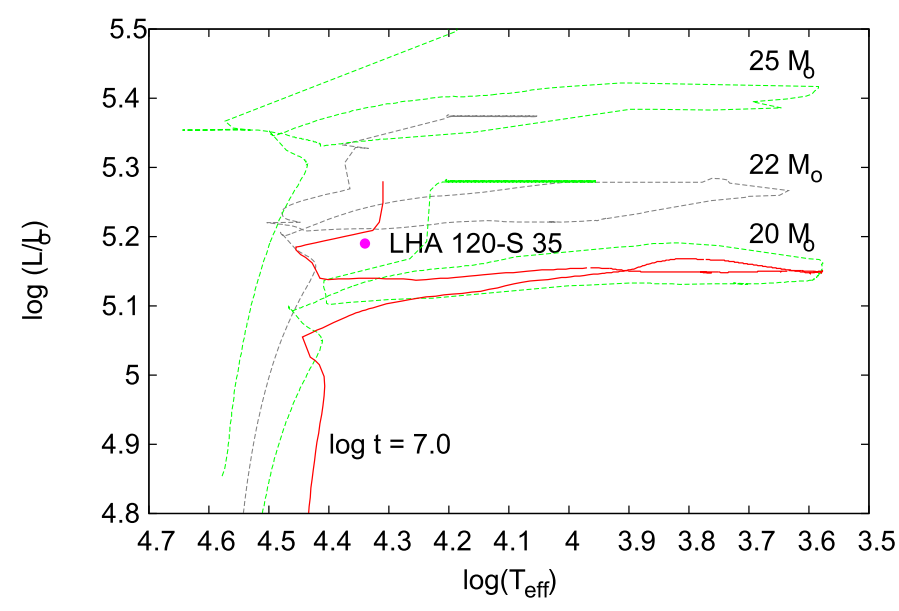

Fig. 23. Hertzsprung-Russell diagram showing the position of LHA 120-S 35. The evolutionary tracks for a rotating massive star with $20 M_{\odot}$ and $25 M_{\odot}$ taken from Ekström et al. (2012) are plotted in green, from which we obtained by interpolation the evolutionary track for a star with $22 M_{\odot}$ (in grey). These tracks correspond to solar metallicity $Z=0.014$. An isochrone of $\log t=7.0$ is shown in red.

LHA 115-S 18, and it is relevant to highlight that it may even disappear at certain times (Torres et al. 2012). Furthermore, the orbital motion in a binary system could also cause variations in the line-profiles. Unfortunately, the data gathered in this work is not enough to make a deep analysis.

Therefore, to unveil the proper scenario for the circumstellar material distribution, high spatial resolution images would be required. However, this is a difficult task, especially for stars located outside the Milky Way, and currently available images from the WISE (Wide-field Infrared Survey Explorer) and Spitzer space telescopes are of too low spatial resolution to provide reasonable information about the environment of
LHA 120-S 35. Figure 22 (top panel) shows a WISE image of a field containing LHA $120-\mathrm{S} 35$, composed by observations obtained with three of the four infrared detectors aboard the satellite (Wright et al. 2010). The composite image shows in blue the infrared light at $4.6 \mu \mathrm{m}$ (W2 band), which is mainly from background stars. The light at $12 \mu \mathrm{m}$ (W3 band) and $22 \mu \mathrm{m}$ (W4 band), arising primarily from warm dust, is represented by green and red, respectively. Figure 22 (bottom panel) shows a 2 MASS $K$-band image, revealing the presence of a neighbouring star of LHA 120-S 35 at 12 arcsec separation, Sk-66 98, which is unresolved on the WISE image. LHA 120-S 35 has been identified with the infrared source SL 482-IR1 that was classified by van Loon et al. (2005) as a hot and dusty object with a $T_{\text {eff }}=20000 \mathrm{~K}$ and $\log \left(L / L_{\odot}\right)=5.19$. These authors consider SL 482-IR1 as a member of the young stellar cluster SL 482 (also named as LMC 1015) with a cluster age of $\log t=7.2 \pm$ 0.24 (van Loon et al. 2005). Other authors estimated different values for the cluster age ranging from $\log t=7.6$ to 8.0 (Glatt et al. 2010; Ko et al. 2013; Bitsakis et al. 2017). Comparing the position of LHA 120-S 35 in the Hertzsprung-Russell diagram to the evolutionary track of a massive star with $\sim 22 M_{\odot}$ (Ekström et al. 2012), we corroborated that an isochrone of $\log t=7.0$ can reconcile the cluster age derived by van Loon et al. (2005) with the position of a post-RSG object (see Fig. 23). However, this result needs to be taken with caution, since the tracks computed by Ekström et al. (2012) consider solar metallicity. So, for a low metallicity LMC star this scenario can be different. On the other hand, van Loon et al. (2005) also reported that SL 482-IR1 has IR colours similar to other low-excitation planetary nebulae, and they modelled the IR spectral energy distribution assuming an oxygen-rich silicate dust shell envelope with a $T_{\text {dust }}=750 \mathrm{~K}$, which might be detached. However, both the B[e]SGs and the planetary nebulae are in the same locus on the $J-H / H-K_{\text {s }}$ colour-colour diagram (Oksala et al. 2013; Reid 2014), which makes it difficult to assign a classification to the star based on 
its infrared colours. But, as the luminosity of LHA 120-S 35 is higher than the one expected for low-excitation planetary nebulae in the MCs (Kaler \& Jacoby 1991), we can discard its classification as a planetary nebula. Therefore, if we consider the classification of SL 482-IR1 as a B[e]SG and the age inferred by van Loon et al. (2005), LHA 120-S 35 would be the third $\mathrm{B}$ [e]SG known up to now belonging to a young stellar cluster. Recently, the LHA 120-S 35 membership to the cluster SL 482 has been discussed by Bitsakis et al. (2017), who were able to determine the members of the star cluster using a robust decontamination technique, providing a probability of more than $92 \%$ that LHA $120-\mathrm{S} 35$ is member of the cluster. The previously reported $\mathrm{B}[\mathrm{e}] \mathrm{SG}$ associated to open clusters are: LHA 120-S 111, which belongs to the compact cluster NGC 1994 of the LMC (Melgarejo et al. 2001; Lortet \& Testor 1988) and Wd1-9, which is a member of the massive Galactic stellar cluster Westerlund 1 (Clark et al. 2013b; Fenech et al. 2017). The small number of $\mathrm{B}[\mathrm{e}] \mathrm{SG}$ s found in stellar clusters is reasonable taking into account that their lifetime in this post-main-sequence phase is relatively short.

Finally, to determine the proper rotation velocity of the different gas tracers and to study the evolution of the inhomogeneities along the circumstellar structure as well as the origin of the variability of the absorption line-profiles, high-resolution observations regularly taken are needed.

Acknowledgements. We thank the referee Dr. Simon Clark for his valuable comments that helped to improve the manuscript. This publication makes use of the NASA Astrophysics Data System (ADS), the SIMBAD database, operated at CDS, Strasbourg, France, and the data products from the Wide-field Infrared Survey Explorer, which is a joint project of the University of California, Lo Angeles, and the Jet Propulsion Laboratory/California Institute of Technology, funded by the National Aeronautics and Space Administration. We would like to thank the observers (S. Ehlerova, A. Kawka) for obtaining FEROS data. Parts of the observations obtained with the MPG $2.2 \mathrm{~m}$ telescope were supported by the Ministry of Education, Youth and Sports project - LG14013 (Tycho Brahe: Supporting Ground-based Astronomical Observations). We also thank R. Venero for fruitful discussions. A.F.T., L.S.C. and M.L.A. acknowledge financial support from the Universidad Nacional de La Plata (Programa de Incentivos G11/137 and Proyecto Promocional de Investigación y Desarrollo G003) and the CONICET (PIP 0177), Argentina. M.K. acknowledges financial support from GAČR (grant number 17-02337S) and from the European Union European Regional Development Fund, project Benefits for Estonian Society from Space Research and Application (KOMEET, 2014 - 2020.4.01.16-0029). GM acknowledges support from CONICYT, Programa de Astronomía/PCI, FONDO ALMA 2014 Proyecto No 31140024. Financial support for the international cooperation of the Czech Republic and Argentina (AVCR-CONICET/14/003) is acknowledged The Astronomical Institute Ondřejov is supported by the project RVO:67985815. This work was also supported by the institutional research funding IUT40-1 of the Estonian Ministry of Education and Research.

\section{References}

Aret, A., Kraus, M., \& Šlechta, M. 2016, MNRAS, 456, 1424

Aret, A., Kolka, I., Kraus, M., \& Maravelias, G. 2017, in The B[e] Phenomenon: Forty Years of Studies, A. Miroshnichenko, S. Zharikov, D. Korčáková, \& M. Wolf, ASP Conf. Ser., 508, 239

Bartlett, E. S., Clark, J. S., Coe, M. J., Garcia, M. R., \& Uttley, P. 2013, MNRAS, 429, 1213

Bitsakis, T., Bonfini, P., González-Lópezlira, R. A., et al. 2017, ApJ, 845, 56

Bonanos, A. Z., Massa, D. L., Sewilo, M., et al. 2009, AJ, 138, 1003

Chita, S. M., Langer, N., van Marle, A. J., García-Segura, G., \& Heger, A. 2008 , A\&A, 488, L37

Cidale, L. S., Borges Fernandes, M., Andruchow, I., et al. 2012, A\&A, 548, A72

Clark, J. S., Miroshnichenko, A. S., Larionov, V. M., et al. 2000, A\&A, 356, 50

Clark, J. S., Bartlett, E. S., Coe, M. J., et al. 2013a, A\&A, 560, A10

Clark, J. S., Ritchie, B. W., \& Negueruela, I. 2013b, A\&A, 560, A11
Conti, P. S. 1997, in Luminous Blue Variables: Massive Stars in Transition, A. Nota \& H. Lamers, ASP Conf. Ser. 120, 161

Crowther, P. A., Lennon, D. J., Walborn, N. R., \& Smartt, S. J. 2006, ArXiv e-print [arxiv: astro-ph/0606717]

Danchi, W. C., Tuthill, P. G., \& Monnier, J. D. 2001, ApJ, 562, 440

Davies, B., Oudmaijer, R. D., \& Sahu, K. C. 2007, ApJ, 671, 2059

de Jager, C. 1998, A\&A Rev., 8, 145

Domiciano de Souza, A., Driebe, T., Chesneau, O., et al. 2007, A\&A, 464, 81

Dunstall, P. R., Fraser, M., Clark, J. S., et al. 2012, A\&A, 542, A50

Ekström, S., Georgy, C., Eggenberger, P., et al. 2012, A\&A, 537, A146

Fenech, D. M., Clark, J. S., Prinja, R. K., et al. 2017, MNRAS, 464, L75

Fukagawa, M., Hayashi, M., Tamura, M., et al. 2004, ApJ, 605, L53

Glatt, K., Grebel, E. K., \& Koch, A. 2010, A\&A, 517, A50

Groh, J. H., \& Vink, J. S. 2011, A\&A, 531, L10

Gummersbach, C. A., Zickgraf, F.-J., \& Wolf, B. 1995, A\&A, 302, 409

Henize, K. G. 1956, ApJS, 2, 315

Jaschek, M., Jaschek, C., \& Andrillat, Y. 1993, A\&AS, 97, 781

Jorgenson, R. A., Kogan, L. R., \& Strelnitski, V. 2000, AJ, 119, 3060

Kaler, J. B., \& Jacoby, G. H. 1991, ApJ, 382, 134

Kim, H., \& Taam, R. E. 2012, ApJ, 759, 59

Ko, Y., Lee, M. G., \& Lim, S. 2013, ApJ, 777, 82

Kraus, M. 2009, A\&A, 494, 253

Kraus, M., Krügel, E., Thum, C., \& Geballe, T. R. 2000, A\&A, 362, 158

Kraus, M., Cidale, L. S., Arias, M. L., Oksala, M. E., \& Borges Fernandes, M. 2014, ApJ, 780, L10

Kraus, M., Cidale, L. S., Arias, M. L., et al. 2016, A\&A, 593, A112

Kraus, M., Liimets, T., Cappa, C. E., et al. 2017, AJ, 154, 186

Lamers, H. J. G. L. M., Zickgraf, F.-J., de Winter, D., Houziaux, L., \& Zorec, J. 1998, A\&A, 340, 117

Levato, H., Miroshnichenko, A. S., \& Saffe, C. 2014, A\&A, 568, A28

Liermann, A., Kraus, M., Schnurr, O., \& Fernandes, M. B. 2010, MNRAS, 408, L6

Lortet, M.-C., \& Testor, G. 1988, A\&A, 194, 11

Magalhaes, A. M. 1992, ApJ, 398, 286

Magalhães, A. M., Melgarejo, R., Pereyra, A., \& Carciofi, A. C. 2006, in Stars with the $\mathrm{B}[\mathrm{e}]$ phenomenon, eds. M. Kraus \& A. S. Miroshnichenko, ASP Conf. Ser., 355, 147

Maravelias, G., Zezas, A., Antoniou, V., \& Hatzidimitriou, D. 2014, MNRAS, 438, 2005

Maravelias, G., Kraus, M., Aret, A., et al. 2017, in The B[e] Phenomenon: Forty Years of Studies, A. Miroshnichenko, S. Zharikov, D. Korčáková, \& M. Wolf, ASP Conf. Ser., 508, 213

Markova, N., \& Puls, J. 2008, A\&A, 478, 823

Massey, P., Neugent, K. F., Morrell, N., \& Hillier, D. J. 2014, ApJ, 788, 83

Mathew, B., Banerjee, D. P. K., Subramaniam, A., \& Ashok, N. M. 2012, ApJ, 753, 13

Melgarejo, R., Magalhães, A. M., Carciofi, A. C., \& Rodrigues, C. V. 2001, A\&A, 377, 581

Millour, F., Meilland, A., Chesneau, O., et al. 2011, A\&A, 526, A107

Oksala, M. E., Kraus, M., Cidale, L. S., Muratore, M. F., \& Borges Fernandes, M. 2013, A\&A, 558, A17

Oudmaijer, R. D., \& Drew, J. E. 1999, MNRAS, 305, 166

Penny, L. R., \& Gies, D. R. 2009, ApJ, 700, 844

Pérez, L. M., Carpenter, J. M., Andrews, S. M., et al. 2016, Science, 353, 1519

Pojmanski, G. 2002, Acta Astron., 52, 397

Reid, W. A. 2014, MNRAS, 438, 2642

Sallum, S., Eisner, J. A., Hinz, P. M., et al. 2017, ApJ, 844, 22

Schwarzenberg-Czerny, A. 1989, MNRAS, 241, 153

Schwarzenberg-Czerny, A. 1996, ApJ, 460, L107

Schwarzenberg-Czerny, A., \& Beaulieu, J.-P. 2006, MNRAS, 365, 165

Shu, F. H. 2016, ARA\&A, 54, 667

Smith, N., Gehrz, R. D., Campbell, R., et al. 2011, MNRAS, 418, 1959

Stahl, O. 2001, in Eta Carinae and Other Mysterious Stars: The Hidden Opportunities of Emission Spectroscopy, eds. T. R. Gull, S. Johannson, \& K. Davidson, ASP Conf. Ser., 242, 163

Tafoya, D., Gómez, Y., \& Rodríguez, L. F. 2004, ApJ, 610, 827

Torres, A. F., Kraus, M., Cidale, L. S., et al. 2012, MNRAS, 427, L80

van Genderen, A. M., \& Sterken, C. 2002, A\&A, 386, 926

van Loon, J. T., Marshall, J. R., \& Zijlstra, A. A. 2005, A\&A, 442, 597

Vink, J. S. 2000, PhD thesis, Universiteit Utrecht, The Netherlands

Wright, E. L., Eisenhardt, P. R. M., Mainzer, A. K., et al. 2010, AJ, 140, 1868

Yudin, R. V. 1996, A\&A, 312, 234

Zickgraf, F.-J., Wolf, B., Leitherer, C., Appenzeller, I., \& Stahl, O. 1986, A\&A, 163,119

Zickgraf, F.-J., Wolf, B., Stahl, O., \& Humphreys, R. M. 1989, A\&A, 220, 206 